OPEN ACCESS

Edited by:

Sukhjiwan Kaur,

Agriculture Victoria, Australia

Reviewed by:

Dawei Xin,

Northeast Agricultural University,

China

Shahin Yazdifar

Agriculture Victoria, Australia

Yongle $\mathrm{Li}$,

University of Adelaide, Australia

*Correspondence:

Piwu Wang

peiwuw@163.com

Aimal Mandoza

ajmal_mandozai@hotmail.com

${ }^{\dagger}$ These authors have contributed

equally to this work

Specialty section:

This article was submitted to

Plant Breeding,

a section of the journal

Frontiers in Plant Science

Received: 02 June 2020 Accepted: 08 July 2021

Published: 28 July 2021

Citation:

Mandozai A, Moussa AA,

Zhang Q, Qu J, Du Y, Anwari G,

Al Amin N and Wang P (2021)

Genome-Wide Association Study

of Root and Shoot Related Traits

in Spring Soybean (Glycine max L.)

at Seedling Stages Using SLAF-Seq.

Front. Plant Sci. 12:568995.

doi: 10.3389/fpls.2021.568995

\section{Genome-Wide Association Study of Root and Shoot Related Traits in Spring Soybean (Glycine max L.) at Seedling Stages Using SLAF-Seq}

\author{
Ajmal Mandozai*t, Abdourazak Alio Moussa ${ }^{\dagger}$, Qi Zhang, Jing Qu, Yeyao Du, \\ Gulaqa Anwari, Noor Al Amin and Piwu Wang* \\ College of Agronomy, Plant Biotechnology Center, Jilin Agricultural University, Changchun, China
}

Root systems can display variable genetic architectures leading to nutrient foraging or improving abiotic stress tolerance. Breeding for new soybean varieties with efficient root systems has tremendous potential in enhancing resource use efficiency and plant adaptation for challenging climates. In this study, root related traits were analyzed in a panel of 260 spring soybean with genome-wide association study (GWAS). Genotyping was done with specific locus amplified fragment sequencing (SLAF-seq), and five GWAS models (GLM, MLM, CMLM, FaST-LMM, and EMMAX) were used for analysis. A total of 179,960 highly consistent SNP markers distributed over the entire genome with an intermarker distance of $2.36 \mathrm{~kb}$ was used for GWAS analysis. Overall, 27 significant SNPs with a phenotypic contribution ranging from 20 to $72 \%$ and distributed on chromosomes $2,6,8,9,13,16$ and 18 were identified and two of them were found to be associated with multiple root-related traits. Based on the linkage disequilibrium (LD) distance of 9.5 $\mathrm{kb}$ for the different chromosomes, 11 root and shoot regulating genes were detected based on LD region of a maximum 55-bp and phenotypic contribution greater than $22 \%$. Expression analysis revealed an association between expression levels of those genes and the degree of root branching number. The current study provides new insights into the genetic architecture of soybean roots, and the underlying SNPs/genes could be critical for future breeding of high-efficient root system in soybean.

Keywords: GWAS, soybean, SLAF-seq, root and shoot related traits, SNPs, genes, qRT-PCR

\section{INTRODUCTION}

Soybean (Glycine max L.) is the third worldwide cultivated crop that plays a significant role in food and industrial production (Tran and Mochida, 2010). Globally, the human population is projected to rise up to $25 \%$ and nearly hit ten billion. Still, the current rate of genetic yield in key crops species is deficient to fulfill upcoming demand (Hickey et al., 2019). The lack of water, lodging and deficiency of nutrients induced by extreme climate will seriously reduce crop production and endanger worldwide food safety (Sinclair, 2011). Soybean is a major oilseed crop in China, growing on around eight million hectares (Zhang et al., 2004); therefore, continuous improvement in soybean quality and yield is vital.

The soybean roots grow from the seed radical, which has three distinct morphologically established components: a primary taproot that drills down into the soil and originates as a radicle 
from a germinating seed which holds the plant in place; fibrous (lateral) roots that spread out throughout the soil, also referred to as secondary roots that emerge from the taproot, and tertiary roots that originate from lateral roots and take up the nutrients and water from soil (Carlson and Lersten, 2004). Over the last century, most soybean breeding efforts have concentrated on the invention and development of aboveground characters such as, end-use quality and yield (Assefa et al., 2018).

Roots, the below ground part of the plant which is obscured from direct observation, received nearly no direct thought for selection and breeding. Thus, root characteristics are rarely considered as selection criteria (Tuberosa and Salvi, 2007), and therefore fewer root related genes have been detected and many genes leading to root development remain unknown in soybean. However, indirect selection via secondary phenotypes, for example nutrient uptake and drought tolerance was used as a proxy to improve root systems (Rosa et al., 2019). Roots play an important role in absorbing water and nutrients as well as providing protection against biotic and abiotic stresses that can directly affect plant growth, above-ground development and final yield (Khan et al., 2016). In crops, root and shoot characteristics are related to environmental stresses like salinity, wind, aluminum toxicity, flood and drought (Keller et al., 1999; Liu et al., 2005b). A well-developed root system is one of the crop protection mechanisms to defend against abiotic stress. Because to their relative stability across different environments, many root related quantitative traits, such as root biomass, root length, and root volume, usually exhibited high heritability (Tian, 1984; Pantalone et al., 1996; Liu et al., 2005b). Besides, the root system is influenced by management, environmental conditions, and is genotype-dependent as plants respond to soil nutrient restriction and water shortage by increasing root biomass and thus increasing the ratio of root to shoot biomass (Bloom et al., 1985; Abdel-Ghani et al., 2013). Moreover, since roots provide the interface between plants and the complex soil environment (Smith and De Smet, 2012), it is advisable to use root improvement as the main purpose of soybean resistance breeding (such as drought, storm, flood, aluminum toxicity, etc.) (Rong et al., 2011).

Due to the challenge in achieving reliable root trait data from the field, characterizing crops like soybean with improved root system characteristics in the field remains still a major defy to current plant biology and, root phenotyping studies are commonly conducted under controlled environments (Rong et al., 2011; Seck et al., 2020; Chen et al., 2021). Besides, some pieces of evidence support that high yielding soybean varieties are supposed to have propitious root systems at the early stages, which may strongly sustain water and nutrients, resulting in increased yield especially under limited water or nutrient availability (Seck et al., 2020; Chen et al., 2021; Dayoub et al., 2021). To date, several Quantitative Trait Loci (QTL) studies have been conducted to locate shoot and root-related trait QTLs in soybean under various conditions of growth, at diverse developmental stages and involving various genetic populations (Rong et al., 2011; Brensha et al., 2012; Manavalan et al., 2015; Prince et al., 2020; Seck et al., 2020; Chen et al., 2021). Rong et al. (2011) in a controlled experiment under hydroponic culture at seedling stage, used a mapping population containing 165 soybean recombinant inbred lines (RILs) derived from the cross between Zhongdou29 and Zhongdou32, and detected 20 QTLs associated with various root and shoot traits located on eight different chromosomes, along with 9 major QTLs on chromosomes 11 and 14. Brensha et al. (2012) in a QTL analysis of root and shoot traits, utilized a RIL population originated from two contrasted parental lines, 'Essex' and 'Forrest', and mapped 12 QTLs distributed on chromosomes 1, 3, 6, 8, 13, 14 , and 18. Those QTLs were associated with multiple root and shoot traits including basal root thickness, lateral root number, maximum root length, root fresh weight (RFW), root dry weight (RDW), shoot fresh weight (SFW), shoot dry weight (SDW), and RFW/SFW, and RDW/SDW ratios. Manavalan et al. (2015) in a study involving 251 BC2F5 backcross inbred lines developed from the cross Dunbar/PI 326582A, and using a well-watered cone system in a growth chamber, identified a major QTL on chromosome 8 (Satt315-I locus) controlling various seedling shoot and root traits including tap root length, lateral root number, shoot length, and shoot dry weight. Recently, using an inter-specific mapping population comprising $184 \mathrm{~F}_{7: 8}$ RILs grown in a cone system, Prince et al. (2020) constructed a high resolution genetic linkage map, and identified 11 putative QTLs associated with several root and shoot traits at V1 stage of soybean growth. The detected QTLs involved five chromosomes (chromosomes 3, 7, 8, 14, and 20) and harbored various candidate genes. Lately, in a trial using 3-weeks old seedlings derived from a cross between K099 and Fendou16 grown under hydroponic conditions, Chen et al. (2021) successfully detected and validated a major QTL associated with primary root length trait located on chromosome 16 between Sat_165 and Satt621 interval markers. Yet, due to low-density markers and large confidence intervals, the localization of these different QTLs was inconsistent among the different findings and further soybean root studies were necessary to detect more chromosomal regions and ultimately identify consistent loci to further screen and identify candidate genes crucial for marker-assisted selection.

Genome-wide association study (GWAS) is an influential technique that helps researchers to determine that polymorphisms are associated with a specific trait in hundreds or thousands of individuals in the genome, enabling repeated SNPs to be checked against specific traits. Today, the latest progress in sequencing technologies and bioinformatics approaches has rendered GWAS an efficient way compared to conventional QTL mapping methods (Thomson, 2014). GWAS is commonly used in recent years to examine the genetic basis of complex traits in main crops such as soybean and rice (Zhao et al., 2011; Zhou et al., 2015; Huang et al., 2016). Prince et al. (2019) discovered many SNPs and candidate genes associated with multiple seedling root architectural traits, including lateral root number, root surface area, and root volume, using a genomewide association study involving 397 soybean accessions. In a comprehensive genome-wide association analysis involving a panel of 137 early maturing soybean lines genotyped via genotyping-by-sequencing and whole-genome sequencing, Seck et al. (2020) characterized twelve root system architecture traits and revealed 10 SNPs explaining 15 to $25 \%$ of the phenotypic 
variation for root total length and primary root diameter. Those QTL regions harbored chromosomes 1, 3, 6, 7, 10, 13, 18, and 19, and contained two putative candidate genes. Rapid progress in Next-Generation Sequencing (NGS) relevant technologies, such as Specific-Locus Amplified Fragment sequencing (SLAF-seq) has generated a huge number of SNPs that provide substantial opportunities for GWAS studies for various traits in different crops (Hake et al., 2017; Pandey et al., 2017; Zhang X. et al., 2017; Zheng et al., 2018). Furthermore, SLAF-seq was proposed as a fast, reliable, highly efficient and cost-effective method to develop large-scale SNP (Sun et al., 2013) and used in many crops like rice, sesame, cucumber, Brassica napus, etc. (Zhang et al., 2013; Xu et al., 2015; Geng et al., 2016; Li Y. et al., 2017). However, to our knowledge, there is no available report about genome-wide studies for root and shoot related traits using SLAF-seq in soybean. In this study, a panel of 260 spring soybean was used to conduct GWAS using SLAF-seq technology to investigate the genetic basis underlying soybean root and shoot related traits at VC, V1 and V2 stages.

The present study aims to (i) examine phenotypic variation of root and shoot related traits in the spring soybean germplasm; (ii) perform GWAS to detect candidate genes and SNPs associated with root and shoot related traits; and (iii) check the expression level of potential candidate genes associated with root and shoot development across stages.

\section{MATERIALS AND METHODS}

\section{Plant Material and Experimental Design}

In this study, a panel of 260 spring soybean germplasm was used, collected from different sites that cover the major distribution areas in China (Supplementary File 1). A greenhouse soil experiment was arranged in a completely randomized design (CRD) with three replications at the department of Agronomy at Jilin Agricultural University $\left(43.8139^{\circ} \mathrm{N}, 125.4066^{\circ} \mathrm{E}\right)$. The settings for the greenhouse were: $28 / 24^{\circ} \mathrm{C}$ temperature, $65 / 85 \%$ relative humidity, and $16 / 8 \mathrm{~h}$ photoperiod day/night, respectively. The natural population seeds were directly seeded in polyvinyl chloride (PVC) pipes $(8 \mathrm{~cm}$ in diameter and $3.2 \mathrm{~mm}$ thick, $25 \mathrm{~cm}$ height) containing a mixture of soil and vermiculite $(2: 1)$, and regularly watered at 5-day intervals in spring 2019.

\section{Phenotypic Evaluation and Root Scanning}

The root and shoot traits were investigated in three vegetative stages VC (after cotyledons and unifoliate leaves have fully Expanded), V1 (Fully developed leaves at unifoliolate nodes) and V2 (Fully developed trifoliate leaf at node above the unifoliolate nodes) (Fehr and Caviness, 1977) corresponding to 7, 14, and 21 days after germination, respectively. Root growth in soybean is well known to show linear growth from the vegetative (V1) to mid-reproductive (R5) growth stages (Rong et al., 2011). The experiments were repeated three times to increase the credibility of root related traits measurements. The seedlings were carefully removed from soil at the specific time-points and rinsed with tap water to remove the soil. The procedure of washing root was conducted carefully to avoid supplementary root injury and losses. Manually evaluated traits were shoot length (SHL), shoot dry weight (SDW), root dry weight (RDW), total plant biomass (TPB), and root dry weight to shoot dry weight ratio (RDW/SDW) (Table 1). Shoot length was manually measured with the help of a ruler from the top tip of the primary leaf to the base of shoot. After shoot measuring each root system was cut from the shoot and roots were individually scanned via a root scanner-based image (Perfection V800; Epson) set to a resolution of 12800 dots per inch (dpi: 5039.37 dots per $\mathrm{cm}$ ) then analyzed using DJ-GX02 software ${ }^{1}$ (Supplementary File 2). Five root morphological traits were recorded using the root analysis software (Table 1). If data collection could not be measured on the same day, seedlings were conserved by submerging roots in $75 \%$ ethanol to avoid further growth of seedlings. For dry weight biomass, root and shoot were collected individually and dried in the oven for $48 \mathrm{~h}$ at $75^{\circ} \mathrm{C}$, to achieve the constant weight of shoot (SDW) and root (RDW) with the help of an electronic scale.

\section{Statistical Analysis}

Statistical analysis of variance (ANOVA) with repeated values for all the above-mentioned traits at each stage in the natural population of spring soybean was carried out to assess the significance of variability amongst the soybean germplasm. The Pearson correlation coefficients among traits and across stages were analyzed to determine the relationship between seedling traits. Based on entry mean, the broad-sense heritability $\left(\mathrm{H}^{2}\right)$ was estimated for each variable from variance estimates using the following equation (Pace et al., 2015):

$$
\begin{gathered}
H^{2}=\frac{\delta_{G}^{2}}{\delta_{P}^{2}}, \quad \delta_{G}^{2}=\left(\frac{M S G-M S E}{r e p}\right), \\
\delta_{P}^{2}=\left(\frac{M S G-M S E}{r e p}\right)+M S E
\end{gathered}
$$

Where, $\delta_{G}^{2}$ is genotypic variance $\delta_{P}^{2}$ is phenotypic variance, MSE is mean square error, MSG is mean square genotype, and rep $($ rep $=3)$ is the number of repetitions per accession. The Mean

${ }^{1}$ www.Dianjiangtech.com

TABLE 1 | Description of traits which collected manually and by DJ-GX02.

\begin{tabular}{lccc}
\hline Trait name & Unit & Symbol & Measurement description \\
\hline Shoot length & $\mathrm{cm}$ & $\mathrm{SL}$ & Manually (using a ruler) \\
Shoot dry weight & $\mathrm{mg}$ & $\mathrm{SDW}$ & Manually (using an electronic scale) \\
Root dry weight & $\mathrm{mg}$ & $\mathrm{RDW}$ & Manually (using an electronic scale) \\
Root dry weight per & $\mathrm{mg}$ & $\mathrm{RDW} / \mathrm{SDW}$ & Manually (RDW divided by SDW) \\
Shoot dry weight & & & \\
Total plant biomass & $\mathrm{mg}$ & $\mathrm{TPB}$ & Manually (RDW plus SDW) \\
Total root length & $\mathrm{cm}$ & $\mathrm{TRL}$ & Measured electronically (DJ-GX02) \\
Surface area & $\mathrm{cm}{ }^{2}$ & $\mathrm{SA}$ & Measured electronically (DJ-GX02) \\
Average diameter & $\mathrm{mm}$ & $\mathrm{AD}$ & Measured electronically (DJ-GX02) \\
Root volume & $\mathrm{cm}^{3}$ & $\mathrm{RV}$ & Measured electronically (DJ-GX02) \\
Branching number & - & $\mathrm{BN}$ & Measured electronically (DJ-GX02)
\end{tabular}


values of each line were used to examine the distribution of each trait across the three stages. All statistical analyses were performed using SPSS Statistics v.21 (IBM Corporation, MO, United States).

\section{Genotyping of Soybean Germplasms}

The 260 Spring Soybean germplasm were sequenced using SLAF-seq technology (Sun et al., 2013; Han et al., 2016) to generate molecular markers throughout the whole genome. The sequencing data have been deposited in the sequence read archive $^{2}$ under the accession number PRJNA681350.

\section{DNA Extraction From Soybean}

All samples were collected from plants growing in the experimental fields at Jilin Agricultural University, Changchun, China. Cetyltrimethylammonium bromide (CTAB) technique (slightly modified), as described by Murray and Thompson (1980) was used for DNA extraction from young and healthy leaves of each soybean accession. NanoDrop Spectrometer (ND-1000 Spectrophotometer, Thermo Scientific) was used to determine the DNA concentration and consistency of all samples. For SLAF sequencing, the quantified DNA was diluted to $100 \mathrm{ng} \cdot \mu \mathrm{l}-1$.

\section{Enzyme Digestion, SLAF Library Preparation, and Sequencing}

In order to acquire more than 309244 SLAF tags (defined as an enzyme fragment sequence of 364-464 bp) per genome, restriction enzyme combinations were tested and selected using in silico digestion prediction using the following criteria: To obtain more than 309244 SLAF tags per genome, combinations of restriction enzymes were examined and selected using the following criteria in the silico digestion prediction (1) The proportion restriction fragments in the repeat sequence was as low as possible; (2) The distribution of enzyme fragments in the genome was as uniform as possible; (3) The complementarity between the length of enzyme fragment and the specific reference genome; and (4) The number of SLAF tags met the expected number. Based on these criteria, RsaI and HaeIII (NEB, Ipswich, MA, United States) were selected as restriction enzymes. To obtain the SLAF tags, DNA from each soybean accession was digested, followed by fragment end reparation, dual-index paired-end adapter ligation, PCR amplification, and target fragment selection for SLAF library construction (Sun et al., 2013). To evaluate the accuracy of the restriction enzymes digestion experiment, rice Nipponbare (Oryza sativa ssp. Japonica) data genome (374.30 MB) (download address ${ }^{3}$ ) was used as a control. Subsequently, the SLAF-seq was performed with an Illumina HiseqTM 2500 (Illumina, Inc., San Diego, CA, United States) at the Biomarker Technologies Corporation. ${ }^{4}$ To obtain the reads for each sample, the raw SLAF-seq data was analyzed using Dual-Index software (Kozich et al., 2013). After filtering out the adapter reads, the sequencing accuracy

\footnotetext{
${ }^{2}$ https://www.ncbi.nlm.nih.gov/sra

${ }^{3}$ http://rapd.dna.affrc.go.jp/

${ }^{4}$ http://Biomarker.com.cn/
}

was evaluated by measuring the guanine-cytosine (GC) content and Q30 $\left(Q=-10 \times \log _{10}(\mathrm{P})\right)$; suggesting a $0.1 \%$ risk of error and therefore $99.9 \%$ confidence). Ultimately, based on the sequence similarity, all SLAF paired-end sample reads were grouped via BLAT software (Kent, 2002). Polymorphic SLAF tags showed sequence polymorphisms between different accessions. High-consistent SLAF tags that show polymorphisms between accessions were then mapped to the soybean reference genome using BWA software (Li and Durbin, 2009).

\section{Identification of SNPs}

SNP loci were identified based on the polymorphic SLAF tags information using primarily GATK (McKenna et al., 2010). Based on clean reads mapped against the reference genome, local realignments were performed, and SNPs were identified using GATK software. For the detailed process, see GATK's official website, https://www.broadinstitute.org/gatk/guide/bestpractices.php. To ensure the reliability of SNPs identified using GATK, SAMtools also was used to detect SNPs with reference to Li et al. (2009). We designated the intersection of SNPs detected by both GATK and SAMtools as the reliable set of SNPs to subject to further analysis. Ultimately, highly consistent SNPs were selected with the criteria of minor allele frequency $(\mathrm{MAF})>0.05$ and marker integrity frequency $>80 \%$ (Zhou et al., 2017) for genetic evolution correlation analysis, linkage disequilibrium analysis, and GWAS analysis.

\section{Population Structure and Linkage Disequilibrium Analysis}

The population structure ( $Q$ matrix) was analyzed using Admixture software (Alexander et al., 2009). The number of subgroups ( $k$-value) was set in from 1 to 15 . The cluster results were cross-validated and the optimum number of subgroups was determined according to the valley value of the cross-validation error rate (Fu and Perry, 2020). A total of 179,960 SNP markers were used to assess the taxonomic and evolutionary relationships between the 260 genotypes via phylogenetic and principal component analyses. To perform the phylogenetic analysis, using the genomic data from the population, the distance between the materials was inferred based on the distance matrix, and then the phylogenetic tree was constructed. Based on SNPs, principal component analysis (PCA) was carried out in order to get the clustering of the different genotypes. The phylogenetic tree was generated using MEGA5 (Tamura et al., 2011) software based on the neighboring method (neighbor-joining), using the Kimura 2-parameter model with bootstrap repeated 1,000 times. PCA was carried out using EIGENSOFT (Price et al., 2006) software. Through Plink2 (Purcell et al., 2007) software, the average decay of LD measured in base pairs was calculated by plotting $\mathrm{r}^{2}$ onto difference between the genetic distance of two base pairs using an $r^{2}$-value of 0.1 as a cut-off.

\section{Genome-Wide Association Studies}

To find out the underlying SNPs or genes associated with root and shoot related traits in a diverse soybean panel, a genome-wide association study was conducted. The association analysis was performed based on the phenotypic dataset and 
the highly consistent population SNPs $(179,960)$. Using the adjusted means for observations on each accession, we used five different statistical GWAS models, GLM, MLM, CMLM of TASSEL 5.0 software (Bradbury et al., 2007), FaST-LMM software (Lippert et al., 2011), and EMMAX software (Kang et al., 2010) to detect the SNPs associated with root and shoot complex traits as well as to analyze their stability across the different stages. Since the Bonferroni correction (BC) for multiple testing (Huang et al., 2010; Yang et al., 2014; Pace et al., 2015) was too conservative and only a few significant SNPs were detected, markers with adjusted $-\log _{10}(P)>4$ (control threshold) and $-\log _{10}(P)>5$ (suggestive threshold) were considered as significant for SNP-trait association. Considering the complexity of root traits, markers that passed the threshold score or above the threshold $-\log _{10}(\mathrm{P})$ was held to be significantly associated with the target trait.

\section{Identification of Candidate Genes and Expression Analysis}

The candidate region of candidate genes was defined by the average LD decay distance or the LD block. High priority candidate genes were chosen based on the criteria that they should have a minimum of $22 \%$ phenotypic contribution and located no more than 55-bp upstream and downstream distance from significant SNPs. For expression analysis, spring soybean lines with extreme phenotypic differences in root branching number were chosen. Thus, four lines with high branching number (Z166, Z201, Z203, and Z232) and four lines with low branching number (Z038, Z049, Z059, and Z063) (Supplementary File 5) were selected among the 260 spring soybean panel as samples for RNA-extraction. The total RNA was isolated from $100 \mathrm{mg}$ root tissues following a Trizol method while the quantity and quality of RNA were examined using a NanoDrop Spectrometer (ND-1000 Spectrophotometer, Thermo Scientific). cDNAs were reverse transcribed from the total RNA using a cDNA kit (All-in-OneTm First-Strand cDNA Synthesis kit, GeneCopoeiaTm) following the manufacturer's standard protocol. The two-step qRT-PCR Thermo cycling conditions were an initial denaturation at $95^{\circ} \mathrm{C}$ for $30 \mathrm{~s}$, followed by 40 cycles of $95^{\circ} \mathrm{C}$ for $5 \mathrm{~s}, 58^{\circ} \mathrm{C}$ annealing for $30 \mathrm{~s}$ and $72^{\circ} \mathrm{C}$ extension for $15 \mathrm{~s}$ and an infinite hold at $10^{\circ} \mathrm{C}$. ActinII was used as internal control gene and the relative expression level of the candidate genes was calculated using the comparative $2^{-\triangle \Delta c t}$ method (Livak and Schmittgen, 2001). All Primer sequences were designed using Primer Premier 5.0 software $^{5}$ (Supplementary File 6).

\section{RESULTS}

\section{Statistical Analysis of Phenotypic Data}

All accessions were examined for root and shoot related traits at 7, 14, and 21 germination days, corresponding to VC, V1, and V2, respectively (Supplementary File 3). Results of analysis of variance (ANOVA) showed substantial variability $(P<0.001)$

\footnotetext{
${ }^{5} \mathrm{http}: / /$ www.premierbiosoft.com
}

among varieties for all the traits evaluated at the three stages (Table 2). The descriptive statistics of all traits for the 260 spring soybean lines are listed in Table 2. High variability among the different traits investigated was observed at all stages. The coefficient of variation (CV) ranged from 7.38 to $36.61 \%$ for all seedling traits at all stages (Table 2). The highest variation was observed for the $\mathrm{BN}$ at VC stage, while the lowest variation was performed by $\mathrm{AD}$ at $\mathrm{V} 1$ stage. The outcome of skewness and kurtosis of all traits at all stages indicate that the distribution of frequencies tended to a normal curve according to the ShapiroWilk test (Supplementary File 4). The broad-sense heritability $\left(\mathrm{H}^{2}\right)$ of root and shoot related traits was estimated, and the values ranged from $27 \%$ for $\mathrm{AD}$ to $86 \%$ for SL, indicating that the observed variation in root related traits is genetically controlled in greenhouse conditions. The Pearson correlation coefficients of all ten seedling root and shoot related traits were also analyzed at each stage, and most of the traits displayed significant positive correlations with similar tendencies at all stages $(P<0.05$, $P<0.01$, Table 3). As shown in Table 3, correlations among the

TABLE 2 | Descriptive statistical results of seedling root and shoot related traits at three stages.

\begin{tabular}{|c|c|c|c|c|c|c|c|c|}
\hline Traits & Stage & Mean & $\pm \mathrm{SD}$ & Skewness & Kurtosis & Range & $\begin{array}{l}\text { CV } \\
(\%)\end{array}$ & $\begin{array}{l}H^{2} \\
(\%)\end{array}$ \\
\hline \multirow[t]{3}{*}{ SL } & VC & 13.87 & 2.48 & -0.01 & -0.57 & 12.00 & 17.88 & 80 \\
\hline & $\mathrm{V}_{1}$ & 19.65 & 2.47 & 0.16 & 0.09 & 14.84 & 12.57 & 8 \\
\hline & V2 & 25.48 & 2.85 & 0.01 & -0.08 & 17.84 & 11.19 & 8 \\
\hline \multirow[t]{3}{*}{ RDW } & VC & 0.03 & 0.01 & 0.08 & -0.58 & 0.05 & 33.33 & \\
\hline & $V_{1}$ & 0.11 & 0.02 & -0.40 & -0.05 & 0.13 & 18.18 & \\
\hline & V2 & 0.20 & 0.03 & 0.36 & 2.84 & 0.26 & 15.00 & 4 \\
\hline \multirow[t]{3}{*}{ SDW } & VC & 0.15 & 0.03 & 0.23 & -0.16 & 0.17 & 20.00 & 6 \\
\hline & V1 & 0.32 & 0.06 & -0.48 & 0.42 & 0.39 & 18.75 & 56 \\
\hline & V2 & 0.51 & 0.07 & 0.08 & 2.27 & 0.57 & 13.73 & 5 \\
\hline RDW/ & VC & 0.23 & 0.06 & 0.33 & 0.46 & 0.40 & 26.00 & 3 \\
\hline \multirow[t]{2}{*}{ SDW } & V1 & 0.35 & 0.06 & 0.56 & 0.73 & 0.31 & 17.14 & 4 \\
\hline & V2 & 0.39 & 0.05 & 0.18 & 1.17 & 0.36 & 12.82 & 5 \\
\hline \multirow[t]{3}{*}{ TPB } & VC & 0.18 & 0.04 & 0.21 & -0.21 & 0.22 & 22.22 & \\
\hline & $\mathrm{V}_{1}$ & 0.43 & 0.08 & -0.53 & 0.51 & 0.52 & 18.60 & \\
\hline & V2 & 0.71 & 0.09 & 0.05 & 2.96 & 0.81 & 12.68 & 4 \\
\hline \multirow[t]{3}{*}{ TRL } & VC & 193.35 & 53.95 & 0.15 & -0.53 & 248.81 & 27.90 & 5 \\
\hline & V1 & 405.34 & 68.29 & -0.41 & 0.09 & 380.43 & 16.85 & 4 \\
\hline & V2 & 582.49 & 75.81 & 0.44 & 0.54 & 424.67 & 13.01 & 38 \\
\hline \multirow[t]{3}{*}{ SA } & VC & 72.14 & 21.4 & 0.10 & -0.57 & 103.09 & 29.66 & 68 \\
\hline & V1 & 157.67 & 27.23 & -0.22 & -0.08 & 147.82 & 17.27 & 4 \\
\hline & V2 & 241.06 & 32.72 & -0.03 & 0.60 & 206.12 & 13.57 & 4 \\
\hline \multirow[t]{3}{*}{$A D$} & VC & 1.18 & 0.1 & 0.07 & 0.08 & 0.57 & 8.47 & 2 \\
\hline & V1 & 1.22 & 0.09 & 0.02 & 0.06 & 0.54 & 7.38 & 27 \\
\hline & V2 & 1.31 & 0.14 & -0.09 & 0.60 & 1.02 & 10.69 & 50 \\
\hline \multirow[t]{3}{*}{ RV } & VC & 2.14 & 0.69 & 0.22 & -0.60 & 3.36 & 32.24 & 61 \\
\hline & V1 & 4.82 & 1.07 & 0.06 & 0.14 & 5.62 & 22.20 & \\
\hline & V2 & 7.94 & 1.58 & 0.36 & 1.35 & 11.43 & 19.90 & \\
\hline \multirow[t]{3}{*}{$\mathrm{BN}$} & VC & 129.07 & 47.25 & 0.33 & -0.36 & 223.00 & 36.61 & 63 \\
\hline & V1 & 243.99 & 61.69 & 0.40 & 0.51 & 408.33 & 25.28 & 63 \\
\hline & V2 & 430.29 & 81.49 & 0.68 & 1.70 & 543.00 & 18.94 & 6 \\
\hline
\end{tabular}

$S L=$ shoot length; SDW = shoot dry weight; $R D W=$ root dry weight; $T R L=$ total root length; $A D=$ average diameter; RDW/SDW = root dry weight per shoot dry weight; TPB = total plant biomass; $R V=$ root volume; $S A=$ surface area; $B N=$ branching number; $S D=S t d$. dev; $C V=$ coefficient of variation; $H^{2}=$ Broad-sense heritability. 
TABLE 3 | Pearson correlation coefficients among all seedling root and shoot traits at three stages.

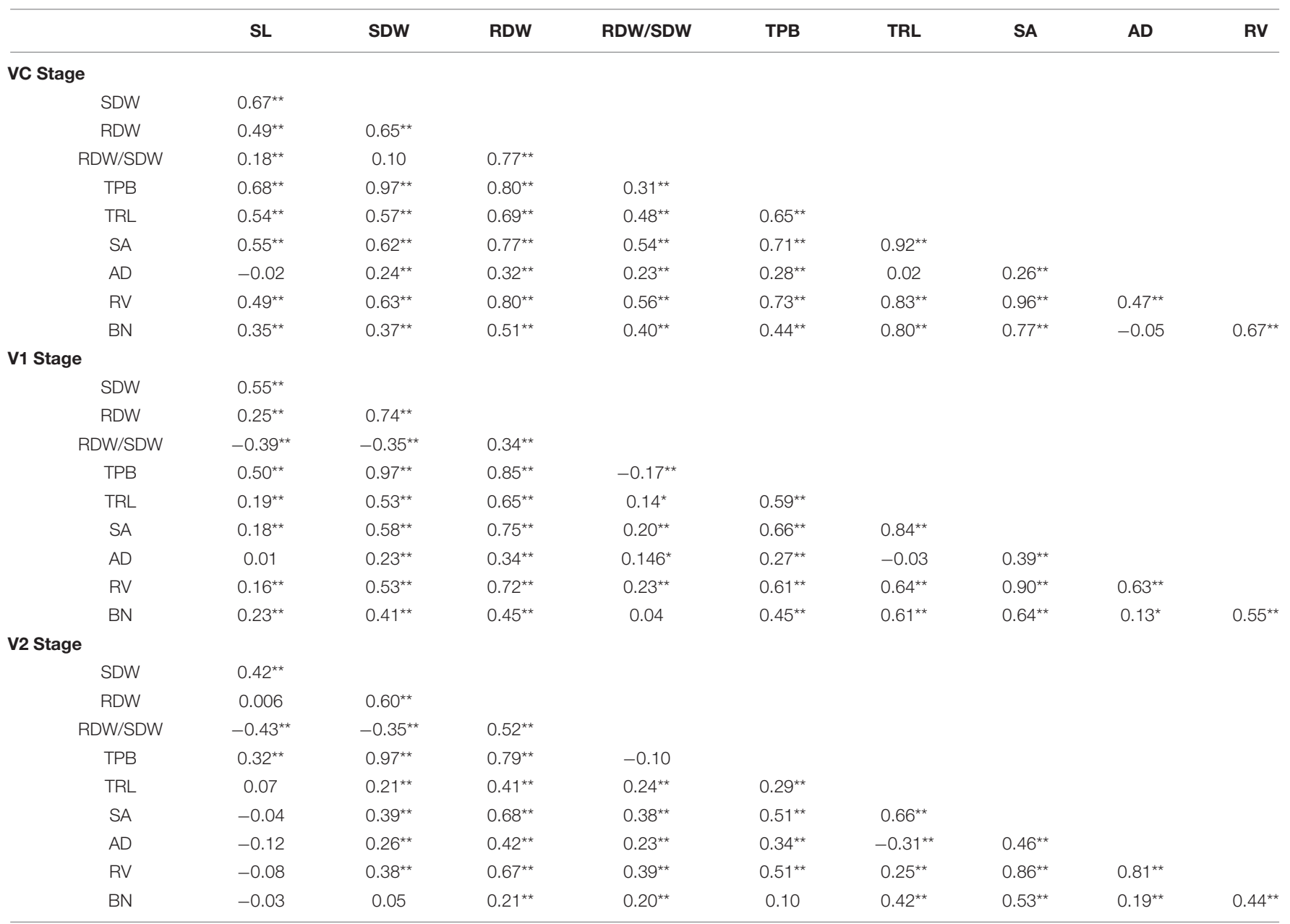

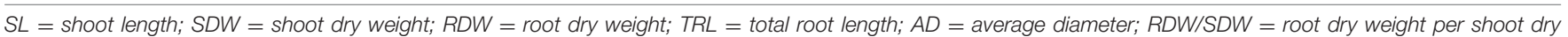
weight; TPB = total plant biomass; $R V=$ root volume; $S A=$ surface area; $B N=$ branching number, * significant at $P<0.05$ and ${ }^{* *}$ significant at $P<0.01$.

following six seedling root and shoot related traits, TRL, RDW, SDW, TPB, RV, and SA were especially high and positive at all the three stages. The highest positive significant correlation was found between TPB and SDW (0.97) and the lowest one between TRL and RDW/SDW $(0.12)(P<0.01$ or $P<0.05$, Table 3$)$. Interestingly, the TRL and RDW were highly correlated with root SA at all stages $(0.66-0.92,0.68-0.77$, respectively). In addition, positive and significant correlations were found between TPB, RDW, and SDW $(r>0.80)$ as well as between RV and SA $(r>0.86)$ at all stages (Table 3$)$.

\section{Genotyping Analysis}

A total of $1263.91 \mathrm{Mb}$ reads were obtained, and the cutting efficiency of RsaI-HaeIII (restriction enzyme for digestion) was $85.65 \%$. The averages Q30 and GC content were 94.01 and $39.87 \%$, respectively. The number of tags was recorded on the 20 soybean chromosomes (Supplementary File 8). Bioinformatics analyses revealed 1,102,987 SLAF tags, of which 609,361 polymorphic, and 2,311,337 SNP markers. Upon filtering the genotype results for minimum MAF of 0.05 and locus integrity of 0.8 , a total of 179,960 highly consistent SNPs was obtained for GWAS, population structure and LD analyses, making the SNP density along the 20 chromosomes one SNP per $2.36 \mathrm{~kb}$ (Figure 1).

\section{Population Structure and Linkage Disequilibrium}

Based on the results of the cross-validation (CV) error rate and $K$-values from Admixture analysis, the 260 genotypes were subdivided into ten subgroups $(K=10$, associated with the lowest $\mathrm{CV}$ error) originated from two ancestral lines (Figures 2A,B). These results have furthermore been validated by the phylogenetic tree and principal components analysis. From the phylogenetic tree, it can be concluded that the soybean lines originated from two large branches which suggests that the 260 soybean lines were from the same ancestors (Figure 2D). However, in the process of evolution, they evolved in different subgroups. By transforming a set of correlated variables into a set of linearly uncorrelated variables, PCA allows different subgroups to be clustered based on the degree of SNP difference of different materials. According to the PCA results, the 260 soybean lines 

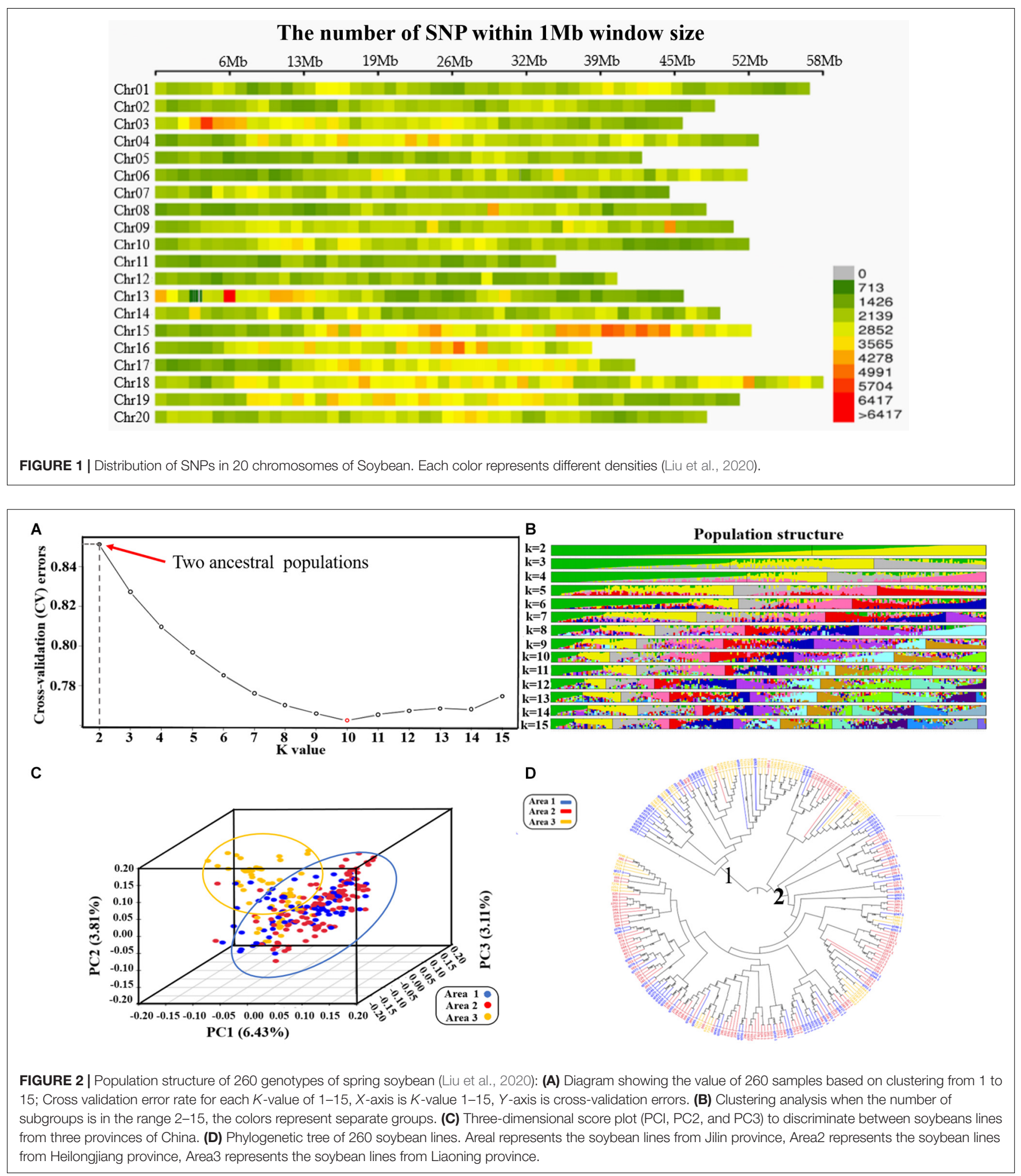

can be divided into different subgroups with more overlapping areas. PC1, PC2, and PC3 accounted for 6.43, 3.81, and 3.11\% respectively (Figure 2C). Collectively, it can be concluded that the 260 soybean lines were subdivided into 10 subgroups represented as an admixture of two ancestral populations. By spanning across all 20 chromosomes a subset of high-quality markers (see section "Materials and Methods"), we evaluated genome-wide LD in the 260 soybean accessions. The average decay distance of $\mathrm{LD}$ for all 
the 260 soybean lines was approximately $9.5 \mathrm{~kb}$ at a threshold of $r^{2}=0.3$ (Figure 3).

\section{Genome-Wide Association Analysis}

In this analysis, as the multiple testing for $\mathrm{BC}$ was too conservative, to further confirm the significant SNPs associated with root related traits, all the 260 spring soybean genotypes were analyzed at three various stages using five GWAS models (GLM, MLM, CMLM, FaST-LMM, and EMMAX) in order to control false positives. According to the results of GWAS, a total of 27 unique SNP markers were detected which exhibited significant associations with root and shoot morphological traits at the critical threshold of $-\log _{10}(P) \geq 4.0$, and these SNP positions were distributed in nine chromosomes, 2, 4, 6, 8, 9, 13, 16, 18, and 20 (Table 4). Minor allele frequencies (MAF) and phenotypic variation ranged from 5 to $47 \%$ and 20 to $72 \%$, respectively, for all the identified SNPs (Table 4). We further analyzed significant SNPs that were repeatedly detected throughout the different stages and methods. In particular, 16 SNPs were consistently detected from two stages while 9 SNPs were detected by at least three GWAS models. These SNPs may therefore be considered major SNPs in this study. Comparing the results from the five GWAS approaches, 22 SNPs were detected by FaST-LMM, 15 SNPs by GLM, 14 SNPs by EMMAX, 7 SNPs by MLM, and 1 SNP by CMLM (Figure 4A and Table 4). The FaST-LMM detected the highest number of significant SNPs, with 22 SNPs detected for the ten traits, while CMLM was the least efficient by detecting only 1 SNP associated with RDW/SDW (Table 4). Besides, FaST-LMM combined with EMMAX followed by FaST-LMM combined with GLM were the combinations that detected the most number of SNPs with, 14 and 10 SNPs, respectively (Figure 4B). Interestingly, several SNPs were simultaneously detected by multiple GWAS models. The SNP with the lowest $p$-value, located on chromosome
16, position $29621947\left(-\log _{10}(P)=7.97, R^{2}=72 \%\right)$, was associated with RDW/SDW and was detected across three different methods (GLM, MLM and CMLM) at VC stage. The second most important SNP found on Chromosome 13, location $40092616\left(-\log _{10}(P)=5.89, R^{2}=34 \%\right)$ was associated with SDW and was detected across two different methods (GLM and MLM) at V2 stage. In regards of SNPs controlling multiple traits, two significant SNPs (Chr13-30887365, Chr09$40442312,-\log _{10}(P)=4.64,5.15$, respectively) were found to be substantially associated with five specific seedling root and shoot traits namely AD, RDW, RV, TPB and SDW (Table 4). Individually, nine SNPs were significantly associated with SDW on chromosome 9 and 13, six SNPs were associated with SA on chromosome 4, 6, 13 and 20, four SNPs were associated with RV on chromosome 13 and 20, and three SNPs were associated with RDW on chromosome 9 and 14, three SNPs were associated with TPB on chromosome 8 and 13 (Table 4). The remaining five SNP positions were related to SL, BN and RDW/SDW along four different chromosomes 2, 9, 16, and 18 (Table 4). Additionally, Q-Q (quantile-quantile) plots and individual Manhattan plots of all the significant SNPs associated with all traits at all stages are shown in Supplementary Files 9, 10, respectively.

\section{Mining of Candidate Genes and Expression Analysis}

In this analysis, eleven candidate genes located within \pm 55 bp distance from the SNPs with phenotypic contribution rate $\left(R^{2}>22 \%\right)$ were obtained as putative candidate genes for root and shoot development in the spring soybean panel. The candidate genes were Glyma.09G179600, Glyma.08G060300, Glyma.16G138900, Glyma.16G208400, Glyma.09G153400, Glyma.13G303800, Glyma.08G060600, Glyma.16G173300, Glyma.02G113900, Glyma.06G148800, and Glyma.18G094200

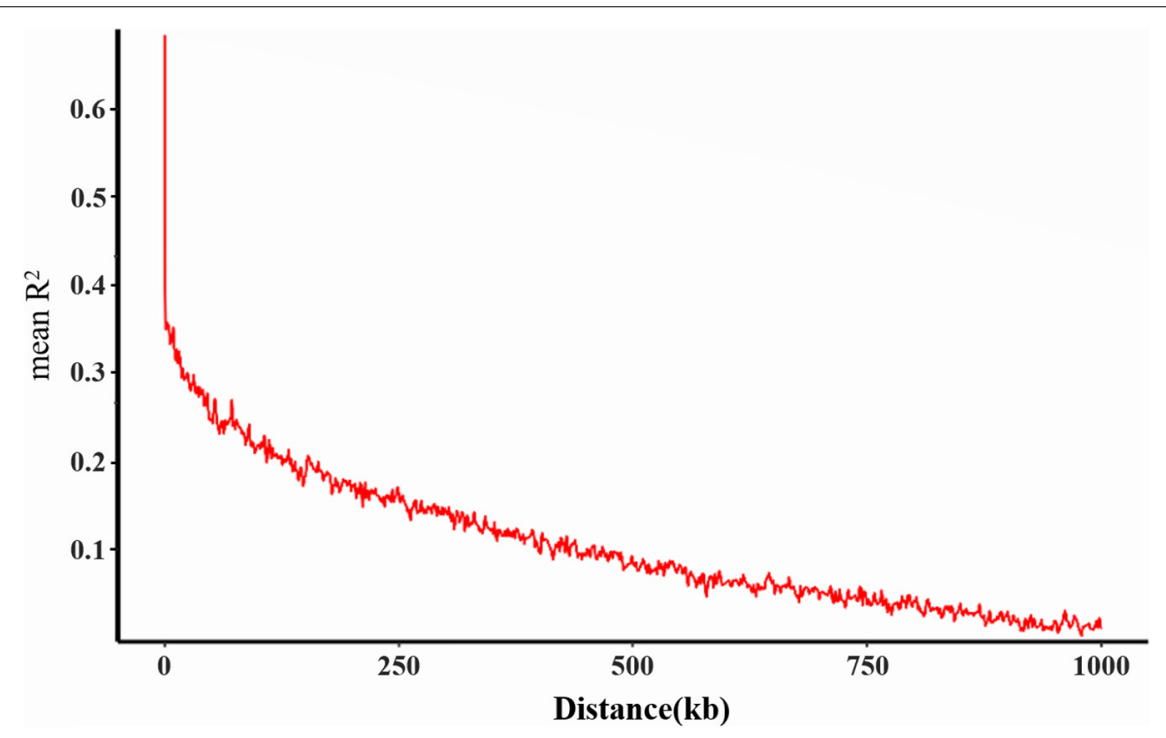

FIGURE 3 | Genome-wide linkage disequilibrium (LD) decay for all 260 accessions. $\mathrm{R}^{2}$ indicates the squared allele frequency correlations between all pairs of SNP markers. The $X$-axis indicates the distance between marker pairs. 
TABLE 4 | Significant SNPs associated with root and shoot related traits detected by GWAS.

\begin{tabular}{|c|c|c|c|c|c|c|c|c|c|c|}
\hline Trait & Position & Method $^{a}$ & Stage $^{b}$ & Chr & $P$-value & $-\log _{10}(P)$ & $\mathbf{R}^{2}$ & Allele & Count & MAF \\
\hline TPB & 4653989 & 1 & 1 & Chr08 & 8.10E-05 & 4.09 & 0.251 & $\mathrm{~T} / \mathrm{C}$ & T:116_C:22 & 0.16 \\
\hline $\mathrm{TRL}$ & 4673257 & 4 & 1 & Chr08 & 3.70E-05 & 4.43 & 0.252 & $\mathrm{C} / \mathrm{G}$ & C:123_G:21 & 0.15 \\
\hline $\mathrm{BN}$ & 9585410 & 1 & 3 & Chr18 & 1.22E-05 & 4.92 & 0.301 & $\mathrm{G} / \mathrm{C}$ & G:110_C:6 & 0.05 \\
\hline $\mathrm{BN}$ & 11071047 & 5,4 & 2 & ChrO2 & $6.17 \mathrm{E}-05$ & 4.21 & 0.300 & $\mathrm{G} / \mathrm{C}$ & G:100_C:18 & 0.15 \\
\hline SA & 12157467 & 4,5 & 3 & Chr06 & 2.18E-05 & 4.66 & 0.323 & $\mathrm{~T} / \mathrm{C}$ & T:79_C:65 & 0.45 \\
\hline SA & 12684509 & $4,1,5$ & 2,3 & Chr04 & $9.8 \mathrm{E}-05$ & 4.01 & 0.238 & $\mathrm{~A} / \mathrm{T}$ & A:93_T:45 & 0.33 \\
\hline RDW/SDW & 29621947 & $2,3,1$ & 1 & Chr16 & 1.08E-08 & 7.97 & 0.721 & C/A & C:113_A:15 & 0.12 \\
\hline SDW & 30444845 & $2,1,4,5$ & 2,3 & Chr13 & 7.1E-05 & 4.15 & 0.260 & $\mathrm{~T} / \mathrm{C}$ & T:130_C:8 & 0.06 \\
\hline SDW & 30444857 & $2,1,4,5$ & 2,3 & Chr13 & 7.1E-05 & 4.15 & 0.260 & $\mathrm{G} / \mathrm{A}$ & G:130_A:8 & 0.06 \\
\hline SDW & 30444862 & $5,1,2,4$ & 2,3 & Chr13 & $1.2 \mathrm{E}-05$ & 4.92 & 0.240 & $A / G$ & A:130_G:8 & 0.06 \\
\hline SDW & 30444865 & $4,1,2,5$ & 2,3 & Chr13 & 7.4E-06 & 5.13 & 0.354 & $\mathrm{C} / \mathrm{T}$ & C:130_T:8 & 0.06 \\
\hline SDW & 30444924 & $1,2,4,5$ & 2,3 & Chr13 & 9.6E-05 & 4.02 & 0.207 & $\mathrm{C} / \mathrm{T}$ & C:130_T:8 & 0.06 \\
\hline RV, SDW, TPB & 30887365 & 4,5 & 2,3 & Chr13 & 2.3E-05 & 4.64 & 0.234 & $\mathrm{~A} / \mathrm{T}$ & A:123_T:11 & 0.08 \\
\hline $\mathrm{RV}$ & 30910036 & 1 & 2,3 & Chr13 & $6.1 \mathrm{E}-05$ & 4.22 & 0.210 & $\mathrm{C} / \mathrm{T}$ & C:114_T:18 & 0.14 \\
\hline SA & 30910036 & 5,4 & 2,3 & Chr13 & $5.2 \mathrm{E}-05$ & 4.29 & 0.212 & $\mathrm{C} / \mathrm{T}$ & C:114_T:18 & 0.14 \\
\hline $\mathrm{RV}$ & 30910036 & $4,1,5$ & 2,3 & Chr13 & $6.1 \mathrm{E}-05$ & 4.07 & 0.253 & $\mathrm{C} / \mathrm{T}$ & C:114_T:18 & 0.14 \\
\hline SL & 31398594 & 1,4 & 2,3 & Chr09 & 7.8E-05 & 4.11 & 0.228 & $\mathrm{~T} / \mathrm{C}$ & T:118_C:8 & 0.06 \\
\hline TRL & 33383414 & 4 & 2 & Chr16 & 2.08E-05 & 4.68 & 0.213 & $\mathrm{G} / \mathrm{T}$ & G:76_T:68 & 0.47 \\
\hline RDW/SDW & 36801463 & 5,4 & 1 & Chr16 & $1.26 \mathrm{E}-05$ & 4.90 & 0.253 & $\mathrm{~T} / \mathrm{A}$ & T:133_A:9 & 0.06 \\
\hline SDW & 37605828 & 4 & 2 & Chr09 & 9.04E-05 & 4.04 & 0.250 & $\mathrm{C} / \mathrm{T}$ & C:76_T:64 & 0.46 \\
\hline SDW & 40092616 & 1,2 & 3 & Chr13 & 1.30E-06 & 5.89 & 0.345 & $\mathrm{C} / \mathrm{G}$ & C:105_G:31 & 0.23 \\
\hline AD, RDW, SDW, TPB & 40442312 & 1,4 & 1 & Chr09 & 7.10E-06 & 5.15 & 0.306 & $\mathrm{C} / \mathrm{A}$ & C:131_A:7 & 0.05 \\
\hline RDW & 40958088 & $1,4,5$ & 2,3 & ChrO4 & 9.7E-05 & 4.01 & 0.264 & $\mathrm{~A} / \mathrm{G}$ & A:117_G:7 & 0.06 \\
\hline SA & 42374764 & 4 & 2,3 & Chr20 & $6.1 \mathrm{E}-05$ & 4.22 & 0.270 & $\mathrm{G} / \mathrm{T}$ & G:124_T:8 & 0.06 \\
\hline SA & 42374776 & 4 & 2,3 & Chr20 & 7.2E-05 & 4.14 & 0.250 & $\mathrm{~A} / \mathrm{T}$ & A:124_T:8 & 0.06 \\
\hline SA & 42374808 & 4 & 2,3 & Chr20 & $7.2 \mathrm{E}-05$ & 4.14 & 0.250 & $\mathrm{~T} / \mathrm{C}$ & T:124_C:8 & 0.06 \\
\hline RV & 45819633 & 4,5 & 2,3 & Chr20 & 7E-05 & 4.16 & 0.251 & $A / G$ & A:105_G:23 & 0.18 \\
\hline
\end{tabular}

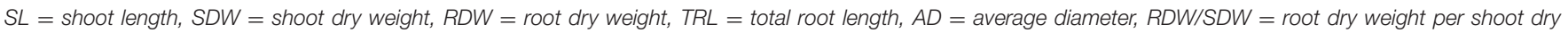

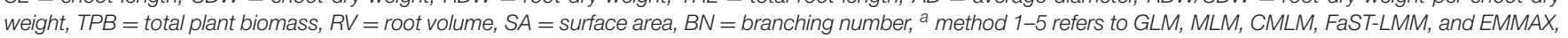
respectively, ${ }^{b}$ stage $1-3$ refers to $V C, V 1$, and V2, respectively, MAF $=$ minor allele frequency, $R^{2}=$ phenotypic contribution, $C h r=$ chromosome.

(Table 5). The list of all genes associated with significant SNPs detected in this study is presented in Supplementary File 7. The functional annotations of these candidate genes were clarified and predicted using NCBI and SoyBase databases (Table 5). The relative expression level results obtained through qRT-PCR revealed that the eleven candidate genes were upregulated at all considered stages (VC, V1, V2) in the different root samples. Comparatively, expression levels in accessions with higher branching number were higher, especially in the later growth stages (Figure 5), indicating that these genes play crucial roles in soybean root development.

\section{DISCUSSION}

Our study's main goal was to elucidate the genetic basis underlying the phenotypic differences in spring soybean root system architecture. Our study described a genome-wide association mapping for root and shoot related traits in a diverse population consisting of 260 natural soybean genotypes. China has preserved $70 \%$ of its unique soybean germplasm in the global gene bank, and many of them are ancient local varieties with high genetic diversity (Qiu et al., 2011). The genetic diversity and widespread phenotypic heterogeneity in such germplasms improve the reliability of GWAS for discovering significant SNPs underlying root traits. Although the optimization of crop root systems has been projected for a long time, genetic dissection and improvement of roots are seldom attempted (Bishopp and Lynch, 2015). Phenotyping of root architecture traits at the adult stage in the field is a complex, costly, and time-consuming task, particularly when a large number of plants need to be phenotyped (Huisman, 1982). Root evaluation methods based on controlled environments such as greenhouse are less timeconsuming, and reliable results easily related to the field can be achieved (Huisman, 1982). Thus, 260 soybean inbred lines were grown under standardized conditions in the greenhouse with three replications in order to get accurate and reliable phenotypes at different seedling stages. The experiment was conducted in three stages (VC, V1, V2) which corresponded to 7, 14, and 21 days after germination (Table 2).

Large phenotypic variations for the ten vegetative period traits can be observed in the 260 spring soybean accessions (Table 2). Our research showed that the phenotypic output of all the investigated root related traits rapidly increased from stage VC to stage V2, supporting the crucial role of the selected stages in root and shoot evaluation in this study. The broad-sense 

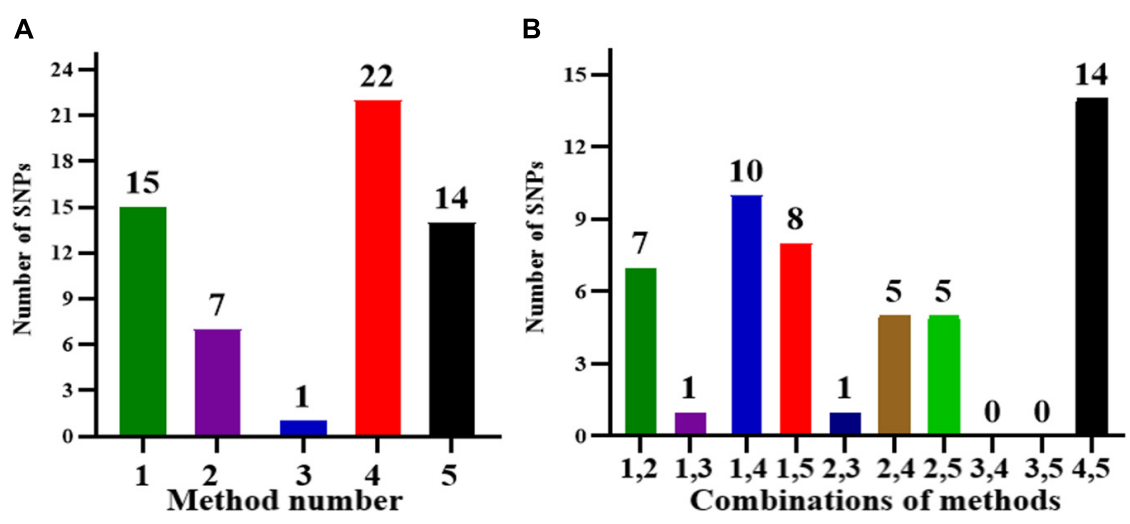

FIGURE 4 | (A) The number of SNPs detected by different methods and (B) different combinations of methods. Method numbers correspond to (1) GLM, (2) MLM, (3) CMLM, (4) FaST-LMM, and (5) EMMAX.

TABLE 5 | Potential candidate genes identified through GWAS and their functional annotations.

\begin{tabular}{|c|c|c|c|c|c|c|}
\hline Genes & Traits & SNP position & Length (bp) & Chr & Distance(bp) & Function explanation \\
\hline Glyma.09G179600 & AD, RDW, SDW, TPB & 40442312 & 2525 & Chr09 & -55 & $\begin{array}{l}\text { PREDICTED: Glycine max aminopeptidase } \\
\text { M1-like (LOC100811437), transcript variant X3, } \\
\text { mRNA }\end{array}$ \\
\hline Glyma.08G060300 & TPB & 4653989 & 2338 & Chr08 & -44 & $\begin{array}{l}\text { PREDICTED: Glycine max uncharacterized } \\
\text { LOC100803004 (LOC100803004), misc_RNA }\end{array}$ \\
\hline Glyma.16G138900 & RDW/SDW & 29621947 & 1464 & Chr16 & -48 & $\begin{array}{l}\text { PREDICTED: Glycine max sugar transporter } \\
\text { ERD6-like 6-like (LOC100801076), mRNA }\end{array}$ \\
\hline Glyma.16G208400 & RDW/SDW & 36801463 & 11522 & Chr16 & 8 & $\begin{array}{l}\text { PREDICTED: Glycine max chloride channel } \\
\text { protein CLC-c-like (LOC100809070), transcript } \\
\text { variant X8, mRNA }\end{array}$ \\
\hline Glyma.09G153400 & SL & 37605828 & 3135 & Chr09 & -41 & $\begin{array}{l}\text { Phaseolus vulgaris hypothetical protein } \\
\text { (PHAVU_009G205900g) mRNA, complete cds }\end{array}$ \\
\hline Glyma.13G303800 & SDW & 40092616 & 5464 & Chr13 & 11 & $\begin{array}{l}\text { PREDICTED: Glycine max G-type lectin } \\
\text { S-receptor-like serine/threonine-protein kinase } \\
\text { At4g03230-like (LOC100817898), mRNA }\end{array}$ \\
\hline Glyma.08G060600 & $\mathrm{TRL}$ & 4673257 & 3725 & Chr08 & 24 & $\begin{array}{l}\text { PREDICTED: Glycine max pentatricopeptide } \\
\text { repeat-containing protein At2g17140-like } \\
\text { (LOC100803535), transcript variant X2, } \\
\text { misc_RNA }\end{array}$ \\
\hline Glyma.16G173300 & $\mathrm{TRL}$ & 33383414 & 832 & Chr16 & -49 & $\begin{array}{l}\text { Glycine max strain Williams } 82 \text { clone } \\
\text { GM_WBb0117F02, complete sequence }\end{array}$ \\
\hline Glyma.02G113900 & $\mathrm{BN}$ & 11071047 & 3810 & ChrO2 & 1 & $\begin{array}{l}\text { PREDICTED: Glycine max tankyrase-2-like } \\
\text { (LOC100795610), transcript variant X1, mRNA }\end{array}$ \\
\hline Glyma.06G148800 & SA & 12157467 & 3573 & Chr06 & -17 & $\begin{array}{l}\text { PREDICTED: Glycine max ribonuclease P protein } \\
\text { subunit p25-like protein-like (LOC100802967), } \\
\text { transcript variant X1, mRNA }\end{array}$ \\
\hline Glyma.18G094200 & $\mathrm{BN}$ & 9585410 & 2581 & Chr18 & 19 & $\begin{array}{l}\text { PREDICTED: Glycine max disease resistance } \\
\text { protein RPM1-like (LOC102663437), mRNA }\end{array}$ \\
\hline
\end{tabular}

heritability of most root related traits was moderate to high (Table 2). In addition, the majority of traits followed a normal distribution indicating the relative stability behind the measured features in this study. Furthermore, all the root related traits were highly significantly and positively correlated at all studied stages. Interestingly, root dry weight (RDW) and total root length (TRL) were extremely correlated with the surface area of root at all stages, which affects the nutrient of plant and water absorption (Comas et al., 2013). In this study, total root length and volume were found tightly correlated. The involvement of total root length and volume in soybean has so far been reported to be associated with aluminum tolerance and drought (Liu et al., 2005b; Yang et al., 2005; Ying et al., 2007). Acquisition of nutrients from soil and accessibility of soil moisture by plants was stated to be more reliant on root length and/or surface area than total root biomass (Nye and Tinker, 1977; Sattelmacher et al., 1990). Also, these traits were previously indicated to regulate the total root growth rate and the root architecture plasticity of plants, which is necessary for successful soil exploration to intercept nutrients and communicate stress signals (Falik et al., 2012). Several studies in 

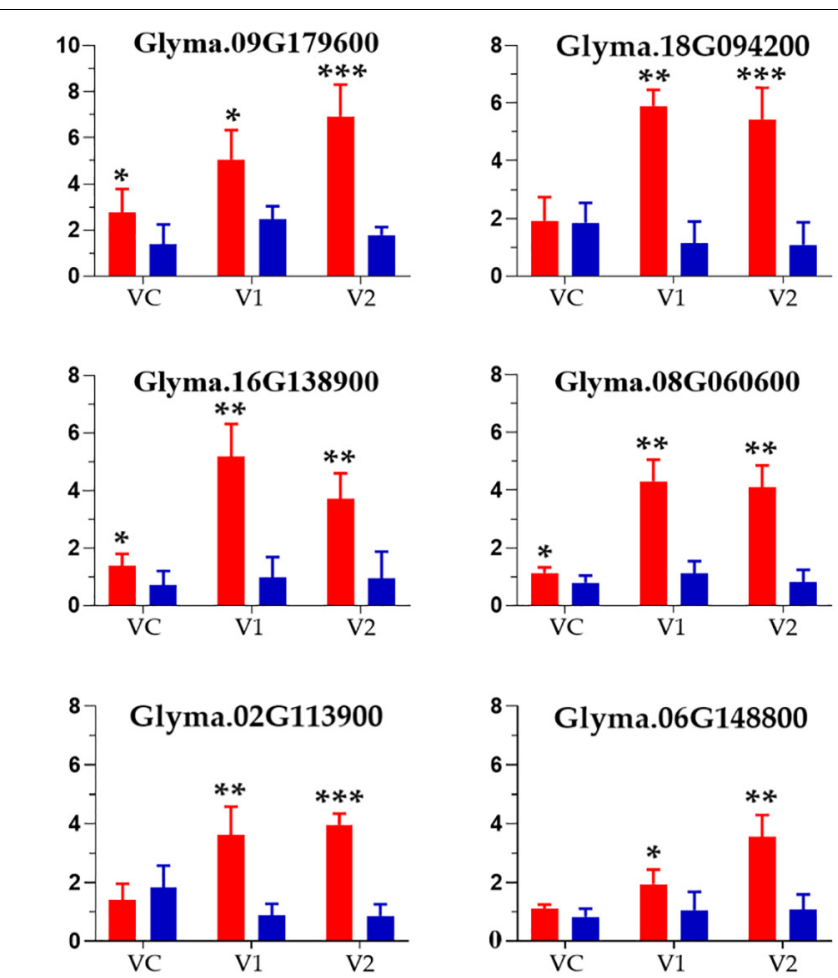
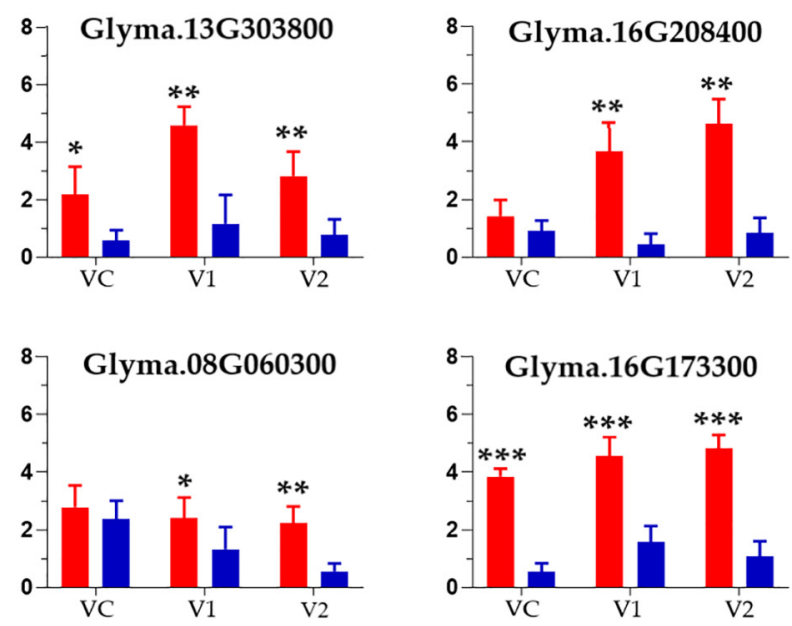

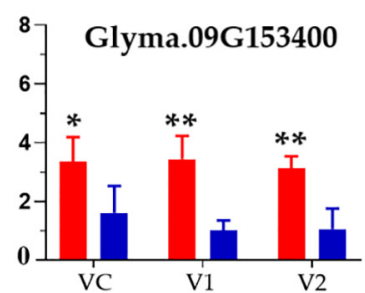

FIGURE 5 | Relative expression levels of eleven candidate genes in phenotypically different root samples from the spring soybean at three seedling stages analyzed via qRT-PCR. The red and blue bars indicate the mean expression level (for four samples) of genes in high and low branching number accessions at VC, VI and V2 stages, respectively; ${ }^{\star * \star},{ }^{* \star}$, and ${ }^{*}$ specify the significance at the level of $P<0.001, P<0.01$, and $P<0.05$, respectively.

crops have emphasized that a deeper root system is influenced by root dry weight and total root length, important for improving drought tolerance and being associated with the final yield under drought stress in soybean (Hudak and Patterson, 1995) and rice (Bengough et al., 2011; Suji et al., 2012). Therefore, identifying ideotypes for root traits involved in drought tolerance at early growth stages could be very useful in guiding the development of soybean cultivars with enhanced soil exploration, and thus water acquisition, under water deficit conditions (Dayoub et al., 2021). In this study, four soybean genotypes including $Z 166$, Z201, Z203 and Z232 with promising root structure were identified. The different genotypes performed better in all the key root architectural traits investigated like, total root length, root branching number, root surface area, root volume, and root dry weight (Supplementary File 5). These accessions are all Chinese landraces, with Z166 from Jiangsu Province and Z201, Z203, and Z232 from Jilin Province (Supplementary File 1).

Understanding the mechanism of root related traits in crops like soybean through SNPs and genes investigation could be the most powerful and successful strategies to develop highquality root cultivars via marker-assisted selection. Many studies have been performed to elucidate the genetic foundation and underlying root genetic function in soybean, and several rootrelated QTLs have been reported in various populations using various environments (Liang et al., 2010, 2014; Lü et al., 2010; Rong et al., 2011). Nevertheless, most of these QTL mapping studies were carried out using low-density genetic linkage maps
(Karikari et al., 2019). In soybean, roots have previously been indicated to be controlled by multigenes, and are strongly affected by environmental changes (Lü et al., 2010). Hence, most of the earlier recorded QTLs were neither stable nor confirmed and have therefore not been successfully utilized in markerassisted selection (MAS) for soybean breeding. To address these limitations, a genome-wide association study based on LD was performed in the present study. Previous studies have shown that GWAS is an effective approach to detecting and identifying SNPs or genes correlated with specific traits, such as root growth in crops (Atwell et al., 2010; Ingvarsson and Street, 2011; Riedelsheimer et al., 2012; Crowell et al., 2016). Based on 179,960 high-quality SNPs in 260 spring soybean accessions, 27 SNPs were identified to be associated with ten root related traits with the significance threshold of $\left(-\log _{10}(P) \geq 4\right)$. Many SNPs found in this analysis were not recorded in earlier studies. This might be clarified by the variance in the genetic background as well as the environmental factors that influence root related traits expression and, ultimately, plant development.

In the present research, besides the most commonly used models, three GWAS models including CMLM, FaST-LMM, and EMMAX were used to improve the accuracy of the results. As GLM uses only Q population structure information while MLM uses $\mathrm{Q}+\mathrm{K}$, due to the strict screening requirements, CMLM and MLM may increase GWAS analysis accuracy. Many researchers have reported that GLM's stringency and accuracy are weaker compared to the MLM (Huang et al., 2010; Yang et al., 2010; 
Zhang et al., 2010; Liu et al., 2016). Furthermore, it had been previously recommended that multiple algorithmic models ought to be used to perform GWAS analysis in real application for complex traits studies (Dhanapal and Crisosto, 2013; Hecht et al., 2013; Zhang H. et al., 2017) due to the limitation in some GWAS scanning models to detect associations with the variation of polygenes. The EMMAX model (Kang et al., 2010) has the ability to handle a large number of markers and reduces the computational time for analysis; FaST-LMM (Lippert et al., 2011) can solve the computational problem but requires that the number of individuals be more than the number of SNPs; and CMLM (Zhang et al., 2010) can boost both statistical power and computing efficiency (speed). Based on these GWAS methods, a different number of SNPs were detected as each method has its specific power and stringency to identify SNP associations. In this study, FaST-LMM followed by GLM were the most effective in detecting more significant SNPs associated with the ten target traits as compared to other models. Thus, 22 SNPs were detected by FaST-LMM, 15 by GLM, 14 by EMMAX, 7 by MLM and 1 by CMLM (Figure 4A and Table 4), and among the combinations of methods, FaST-LMM combined with EMMAX detected the maximum number of SNPs (Figure 4B). Comparatively FaSTLMM detected the greatest number of SNPs followed by GLM, and EMMAX while the small number of SNPs was detected by CMLM and MLM models which confirmed the relevance and ability of the different GWAS models used for controlling the false positives. Several studies used various approaches, along with significant and suggestive level to balance the false negatives and false positives in root related studies (Li X. et al., 2017; Jia et al., 2019). Interestingly, a major SNP, Chr09-40442312 was found to be associated with four root-related traits $\mathrm{AD}, \mathrm{RDW}$, SDW and TPB, and described $30.6 \%$ of the total phenotypic variation. The above-mentioned major SNP overlapped one annotated gene Glyma.09G179600, which encodes an amino acid transport and metabolism. This gene was also previously recorded for soybean nodule-related traits (Li et al., 2018). Thus, this gene may be a promising candidate gene for further study on soybean root development.

Chromosome 13 was found substantially associated with numerous SNPs for multiple root and shoot related traits including RDW, SDW, RV, TPB, and SA. Of these SNPs, Chr1330444845, Chr13-30444857, Chr13-30444862, Chr13-30444865, Chr13-30444924, Chr13-30887365, and Chr13-30910036 overlapped with the reported QTLs 'Root length 1-1', 'Root length 1-2', 'Root length 1-3', 'Root length 1-4', 'Root area $2-1$ ', and 'Root area 2-2' underlying soybean root length and surface area development under hypoxia conditions (Van Nguyen et al., 2017). The soybean salt tolerance loci ss715616115 and ss715624611 on chromosomes 13 (position 33,415,484) and 16 (position 33383414) reported by Zeng et al. (2017) were located especially near the SNPs Ch13-40092616 and Chr16-33383414 associated with SDW and TRL, respectively. SNP Chr13-40092616 was only 11 bp far from the gene model Glyma.13G303800 coding for a G-type lectin S-receptor-like serine/threonine-protein kinase At4g03230-like. This gene was earlier indicated to show high expression in soybean seedling leaves (Libault et al., 2010; Severin et al., 2010). Chromosome
8 harbored SNPs Chr8-4673257 and Chr8-4653989 for two different traits namely TRL and TPB, respectively. SNP Chr8-4673257 was located 24 bp from the candidate gene Glyma.08G060600 predicted to encode a pentatricopeptide repeat-containing protein At2g17140-like highly expressed in nodule roots, leaves and primary root in soybean (Libault et al., 2010; Severin et al., 2010). SNP Chr8-4653989 was located 44 bp downstream the gene model Glyma.08G060300 highly expressed in the soybean primary root and lateral roots (Libault et al., 2010; Severin et al., 2010). Furthermore, several QTLs for root dry weight, taproot length, shoot length, lateral root number, and total root length were previously detected on this linkage group from various similar root studies at seedling stage/time-point (Liu et al., 2005a; Ying et al., 2007; Liang et al., 2014; Manavalan et al., 2015; Prince et al., 2020). Moreover, a region spanning only $15 \mathrm{cM}$ on this chromosome was earlier revealed to contain four cluster QTLs for root fresh weight, root dry weight, shoot fresh weight, and shoot dry weight using the Essex $\times$ Forrest soybean population (Brensha et al., 2012). SNPs Chr6-12157467, Chr18-9585410, and Chr20-45819633 associated with SA, BN, and RV were in LD with three noteworthy QTNs namely q6, q18-2, and q20-3 associated with photosynthetic traits related to phosphorus efficiency in soybean, respectively (Lü et al., 2018). SNP Chr6-12157467 was located 17 bp downstream the gene model Glyma.06G148800 predicted to encode a ribonuclease subunit p25-like protein-like while SNP Chr18-9585410 was $19 \mathrm{bp}$ away from the candidate gene Glyma.18G094200 encoding for a RPM1 protein-like conferring disease resistance. These two genes were recorded to display high expression in soybean primary root (Libault et al., 2010; Severin et al., 2010). Chromosomes 6 and 4 seem to harbor multiple QTLs for soybean root traits. Wang et al. (2004) detected 3 QTLs for root weight on chromosomes 6 with $6.8-26.3 \%$ of the phenotypic contributions at maturity stage of soybean. Liu et al. (2005b) revealed 6 QTLs for drought tolerance on chromosome 6 at V4 stage, including 3 major QTLs explained 22 to $24.7 \%$ of phenotypic variations associated with root dry weight, total root length, and root volume. At R7 stage, Zhou et al. (2009) mapped 1 and 3 QTLs for root weight on chromosomes 6 and 8, respectively, with phenotypic contributions ranged from 8.3 to $12.5 \%$. Our detected SNP Chr06-12157467 associated with SA at V2 stage on chromosome 6 was also found very close to qMRL6, a QTL for main root length linked to the marker locus Sat_153 (10.52 cM), which was earlier detected in a similar study at V2 stage (Rong et al., 2011). In addition, this SNP was in LD with $q M R L / R W 6$, a QTL for main root length to root weight ratio (MRL/RW) linked to marker Satt557 (5.55 cM) (Rong et al., 2011). SNP Chr04-40958088 on chromosome 4 associated with RDW and consistently detected at V1 and V2 stages collocated with qHW4 (Rong et al., 2011), a previously reported QTL for hypocotyl weight between markers Satt476 and Satt294. This SNP was also in LD with a SNP on chromosome 4 (position 36376946) associated with root surface area detected by Prince et al. (2019) at V1 stage. SNPs Chr16-29621947 and Chr16-33383414 on chromosome 16 significantly associated with RDW/SDW and TRL, respectively were in LD with a SNP (position 30021671) for lateral root number and root diameter in thickness Class 
I (Prince et al., 2019). SNPs chr20-42374764, chr20-42374776, chr20-42374808 associated with SA and SNP chr20-45819633 associated with RV were found very close to the SNP (position 43048714) associated with root diameter in volume and surface area Class II (Prince et al., 2019). These loci were also in LD with a QTL for total root length recently identified by Prince et al. (2020) within a SNP06258 NCSB_004833 marker interval.

Based on the LD decay analysis, an approximately 55 bp SNP region was chosen as a candidate region for selecting potential candidate genes. According to the functional annotation, GO enrichment, and gene expression profile, we selected 11 genes for expression analysis using qRT-PCR, widely used to validate GWAS-identified genes expression with high accuracy and sensitivity (Bustin, 2002; Bustin and Nolan, 2004). The relative expression rates of the eleven potential candidate genes tested were significantly higher in high root branching number accessions as compared to low root branching number accessions at all considered growth stages (VC, V1, V2), particularly later stages (Figure 5). Therefore, the evaluated genes served as a constructive regulator in the spring soybean panel which could be of great significance for elite's soybean breeding programs. Upcoming research based on these potential candidate genes will, however thoroughly elucidate the role of these aforementioned genes in soybean root growth and development.

\section{CONCLUSION}

In conclusion, this work provides a comprehensive analysis of the genetic architecture of root related traits in spring soybean. We performed a genome-wide association study through 260 soybean accessions genotyped by SLAF-seq for root related traits at three developmental stages under standard greenhouse conditions and identified a total of 27 substantial SNPs using different GWAS models (GLM, MLM, CMLM, FaST-LMM, and EMMAX). All The detected SNPs showed individual phenotypic contribution rates greater than $20 \%$ and were significantly associated with SDW (7), SA (6), RV (3), TRL (2), BN (2), RDW/SDW (2), TPB (1), RDW (1), and SL (1). However, two major SNPs were found to be associated with multiple traits including AD, RDW, SDW, and TPB in one side and RV, SDW, TPB in the other. Based on the LD region of no more than 55-bp and SNPs with phenotypic contribution rate greater than $22 \%$, eleven promising root and shoot regulating genes viz. Glyma.09G179600, Glyma.08G060300, Glyma.16G138900, Glyma.16G208400, Glyma.09G153400, Glyma.13G303800, Glyma.08G060600, Glyma.16G173300, Glyma.02G113900, Glyma.06G148800, and Glyma.18G094200 overlapping with SNPs on chromosomes 2, 6, 8, 9, 13, 16, and 18 were detected and verified for expression level. The evaluated genes were shown to serve as positive regulators for root branching number in the spring soybean panel, which could play a crucial role in markerassisted selection programs. Thus, SNPs and underlying genes discovered in the current study provide a key basis for revealing the complex genetic mechanism of root development and genetic enhancement of soybean root, as well as supporting the breeding of high-efficiency soybean root system varieties in the future.

\section{DATA AVAILABILITY STATEMENT}

The original contributions presented in the study are publicly available. This data can be found here: https://www.ncbi.nlm.nih. gov/bioproject/PRJNA681350.

\section{AUTHOR CONTRIBUTIONS}

AM drafted the original manuscript. AAM reviewed and edited the manuscript. AM and AAM conducted the experiments and performed the analysis. JQ, QZ, and YD performed the phenotyping. GA and NA conducted formal analysis. PW supervised the experiments. All authors have read and agreed the final version of the manuscript.

\section{FUNDING}

This research was supported by the National Natural Science Foundation of China under grant number (31571689).

\section{ACKNOWLEDGMENTS}

We would like to express our gratitude to Biomarker Company (Beijing, China) for their kind support in SLAF sequencing. We also thank M. I. Nasser for his valuable help to improve this manuscript.

\section{SUPPLEMENTARY MATERIAL}

The Supplementary Material for this article can be found online at: https://www.frontiersin.org/articles/10.3389/fpls.2021. 568995/full\#supplementary-material

Supplementary File 1 | List and provenance of the 260 spring soybean accessions.

Supplementary File 2 | Root trait phenotypic evaluation and scanning

Supplementary File 3 | Phenotypic data of root and shoot related traits of 260 spring soybean population at VC, V1, and V2 stages.

Supplementary File 4 | Histogram of the frequency distribution of root and shoot related traits at all three stages with data from VC stage shown in blue color (A), data from $\mathrm{V} 1$ stage shown in green color (B), and data from V2 stage shown in turquoise color (C).

Supplementary File $\mathbf{5}$ | Accessions used in qRT-PCR analysis.

Supplementary File 6 | List of primers used for the qRT-PCR assay of the investigated genes involved in the root pathway.

Supplementary File 7 | List of all associated genes with significant SNPs detected in this study.

Supplementary File $\mathbf{8}$ | Distribution of SLAF tags and polymorphic SLAF tags on soybean chromosomes.

Supplementary File 9 | Quantile-Quantile (Q-Q) plots of root and shoot related traits at three stages.

Supplementary File 10 | Individual Manhattan plots of all the significant SNPS associated with all traits at all stages. 


\section{REFERENCES}

Abdel-Ghani, A. H., Kumar, B., Reyes-Matamoros, J., Gonzalez-Portilla, P. J., Jansen, C., San Martin, J. P., et al. (2013). Genotypic variation and relationships between seedling and adult plant traits in maize (Zea mays L.) inbred lines grown under contrasting nitrogen levels. Euphytica 189, 123-133. doi: 10.1007/ s10681-012-0759-0

Alexander, D. H., Novembre, J., and Lange, K. (2009). Fast model-based estimation of ancestry in unrelated individuals. Genome Res. 19, 1655-1664.

Assefa, Y., Bajjalieh, N., Archontoulis, S., Casteel, S., Davidson, D., Kovács, P., et al. (2018). Spatial characterization of soybean yield and quality (amino acids, oil, and protein) for United States. Sci. Rep. 8:14653. doi: 10.1038/s41598-01832895-0

Atwell, S., Huang, Y. S., Vilhjálmsson, B. J., Willems, G., Horton, M., Li, Y., et al. (2010). Genome-wide association study of 107 phenotypes in Arabidopsis thaliana inbred lines. Nature 465:627. doi: 10.1038/nature08800

Bengough, A. G., McKenzie, B., Hallett, P., and Valentine, T. (2011). Root elongation, water stress, and mechanical impedance: a review of limiting stresses and beneficial root tip traits. J. Exp. Bot. 62, 59-68. doi: 10.1093/jxb/ erq350

Bishopp, A., and Lynch, J. P. (2015). The hidden half of crop yields. Nat. Plants 1:15117. doi: 10.1038/nplants.2015.117

Bloom, A. J., Chapin, F. S. III, and Mooney, H. A. (1985). Resource limitation in plants-an economic analogy. Annu. Rev. Ecol. Syst. 16, 363-392.

Bradbury, P. J., Zhang, Z., Kroon, D. E., Casstevens, T. M., Ramdoss, Y., and Buckler, E. S. (2007). TASSEL: software for association mapping of complex traits in diverse samples. Bioinformatics 23, 2633-2635. doi: 10.1093/ bioinformatics/btm 308

Brensha, W., Kantartzi, S. K., Meksem, K., Grier, R. L. IV, Barakat, A., Lightfoot, D. A., et al. (2012). Genetic analysis of root and shoot traits in the 'Essex'by 'Forrest'recombinant inbred line (RIL) population of soybean [Glycine max (L.) Merr.]. Plant Genet. Genomics Biotechnol. 1, 1-9. doi: 10.5147/pggb. v1i1.146

Bustin, S. (2002). INVITED REVIEW Quantification of mRNA using real-time reverse transcription PCR (RT-PCR): trends and problems. J. Mol. Endocrinol. 29, 23-39.

Bustin, S. A., and Nolan, T. (2004). Pitfalls of quantitative real-time reversetranscription polymerase chain reaction. J. Biomol. Tech. JBT 15:155.

Carlson, J. B., and Lersten, N. R. (2004). "Reproductive morphology 1," in Soybeans: Improvement, Production, and Uses (Soybeansimprove), eds R. M. Shibles, J. E. Harper, R. F. Wilson, and R. C. Shoemaker (Madison, WI: American Society of Agronomy), 59-95. doi: 10.2134/agronmonogr16.3ed.c3

Chen, H., Kumawat, G., Yan, Y., Fan, B., and Xu, D. (2021). Mapping and validation of a major QTL for primary root length of soybean seedlings grown in hydroponic conditions. BMC Genomics 22:132. doi: 10.1186/s12864-02107445-0

Comas, L., Becker, S., Cruz, V. M. V., Byrne, P. F., and Dierig, D. A. (2013). Root traits contributing to plant productivity under drought. Front. Plant Sci. 4:442. doi: 10.3389/fpls.2013.00442

Crowell, S., Korniliev, P., Falcao, A., Ismail, A., Gregorio, G., Mezey, J., et al. (2016). Genome-wide association and high-resolution phenotyping link Oryza sativa panicle traits to numerous trait-specific QTL clusters. Nat. Commun. 7:10527. doi: $10.1038 /$ ncomms 10527

Dayoub, E., Lamichhane, J. R., Schoving, C., Debaeke, P., and Maury, P. (2021). Early-Stage phenotyping of root traits provides insights into the drought tolerance level of soybean cultivars. Agronomy 11:188. doi: 10.3390/ agronomy11010188

Dhanapal, A. P., and Crisosto, C. H. (2013). Association genetics of chilling injury susceptibility in peach (Prunus persica (L.) Batsch) across multiple years. 3 Biotech 3, 481-490. doi: 10.1007/s13205-012-0109-x

Falik, O., Mordoch, Y., Ben-Natan, D., Vanunu, M., Goldstein, O., and Novoplansky, A. (2012). Plant responsiveness to root-root communication of stress cues. Ann. Bot. 110, 271-280. doi: 10.1093/aob/mcs045

Fehr, W. R., and Caviness, C. E. (1977). Stages of Soybean Development. Special Report 80. Ames, IA: Iowa State University. Agricultural and Home Economics Experiment Station.

Fu, W., and Perry, P. O. (2020). Estimating the number of clusters using crossvalidation. J. Comput. Graph. Stat. 29, 162-173.
Geng, X., Jiang, C., Yang, J., Wang, L., Wu, X., and Wei, W. (2016). Rapid identification of candidate genes for seed weight using the SLAF-Seq method in Brassica napus. PLoS One 11:e0147580. doi: 10.1371/journal.pone.0147580

Hake, A. A., Shirasawa, K., Yadawad, A., Sukruth, M., Patil, M., Nayak, S. N., et al. (2017). Mapping of important taxonomic and productivity traits using genic and non-genic transposable element markers in peanut (Arachis hypogaea L.). PLoS One 12:e0186113. doi: 10.1371/journal.pone.0186113

Han, Y., Zhao, X., Liu, D., Li, Y., Lightfoot, D. A., Yang, Z., et al. (2016). Domestication footprints anchor genomic regions of agronomic importance in soybeans. N. Phytol. 209, 871-884. doi: 10.1111/nph.13626

Hecht, B. C., Campbell, N. R., Holecek, D. E., and Narum, S. R. (2013). Genomewide association reveals genetic basis for the propensity to migrate in wild populations of rainbow and steelhead trout. Mol. Ecol. 22, 3061-3076. doi: $10.1111 / \mathrm{mec} .12082$

Hickey, L. T., Hafeez, A. N., Robinson, H., Jackson, S. A., Leal-Bertioli, S. C. M., Tester, M., et al. (2019). Breeding crops to feed 10 billion. Nat. Biotechnol. 37, 744-754. doi: 10.1038/s41587-019-0152-9

Huang, X., Sang, T., Zhao, Q., Feng, Q., Zhao, Y., Li, C., et al. (2010). Genome-wide association studies of 14 agronomic traits in rice landraces. Nat. Genet. 42:961. doi: 10.1038/ng.695

Huang, X., Yang, S., Gong, J., Zhao, Q., Feng, Q., Zhan, Q., et al. (2016). Genomic architecture of heterosis for yield traits in rice. Nature 537:629. doi: 10.1038/ nature 19760

Hudak, C., and Patterson, R. (1995). Vegetative growth analysis of a droughtresistant soybean plant introduction. Crop Sci. 35, 464-471. doi: 10.2135/ cropsci1995.0011183X003500020031x

Huisman, O. (1982). Interrelations of root growth dynamics to epidemiology of root-invading fungi. Annu. Rev. Phytopathol. 20, 303-327. doi: 10.1146/ annurev.py.20.090182.001511

Ingvarsson, P. K., and Street, N. R. (2011). Association genetics of complex traits in plants. N. Phytol. 189, 909-922. doi: 10.1111/j.1469-8137.2010.03593.x

Jia, Z., Liu, Y., Gruber, B. D., Neumann, K., Kilian, B., Graner, A., et al. (2019). Genetic dissection of root system architectural traits in spring barley. Front. Plant Sci. 10:400. doi: 10.3389/fpls.2019.00400

Kang, H. M., Sul, J. H., Service, S. K., Zaitlen, N. A., Kong, S.-Y., Freimer, N. B., et al. (2010). Variance component model to account for sample structure in genome-wide association studies. Nat. Genet. 42:348.

Karikari, B., Chen, S., Xiao, Y., Chang, F., Zhou, Y., Kong, J., et al. (2019). Utilization of interspecific high-density genetic map of RIL population for the QTL detection and candidate gene mining for 100-seed weight in soybean. Front. Plant Sci. 10:1001. doi: 10.3389/fpls.2019.01001

Keller, M., Karutz, C., Schmid, J., Stamp, P., Winzeler, M., Keller, B., et al. (1999). Quantitative trait loci for lodging resistance in a segregating wheat $\times$ spelt population. Theor. Appl. Genet. 98, 1171-1182. doi: 10.1007/s001220051182

Kent, W. J. (2002). BLAT-the BLAST-like alignment tool. Genome Res. 12, 656-664.

Khan, M., Gemenet, D. C., and Villordon, A. (2016). Root system architecture and abiotic stress tolerance: current knowledge in root and tuber crops. Front. Plant Sci. 7:1584. doi: 10.3389/fpls.2016.01584

Kozich, J. J., Westcott, S. L., Baxter, N. T., Highlander, S. K., and Schloss, P. D. (2013). Development of a dual-index sequencing strategy and curation pipeline for analyzing amplicon sequence data on the MiSeq Illumina sequencing platform. Appl. Environ. Microbiol. 79, 5112-5120. doi: 10.1128/AEM. 01043-13

Li, C., Chang, H., Wang, J., Zou, J., Shi, Y., Zhang, Y., et al. (2018). Genetic analysis of nodule traits in soybean via wild soybean background population (Glycin soja) and high generation recombination inbred lines [Glycine max (L.) Merr.]. Int. J. Agric. Biol. 20, 2521-2528. doi: 10.17957/IJAB/15.0794

Li, H., and Durbin, R. (2009). Fast and accurate short read alignment with Burrows-Wheeler transform. Bioinformatics 25, 1754-1760. doi: 10.1093/ bioinformatics/btp324

Li, H., Handsaker, B., Wysoker, A., Fennell, T., Ruan, J., Homer, N., et al. (2009). The sequence alignment/map format and SAMtools. Bioinformatics 25, 20782079. doi: 10.1093/bioinformatics/btp352

Li, X., Guo, Z., Lv, Y., Cen, X., Ding, X., Wu, H., et al. (2017). Genetic control of the root system in rice under normal and drought stress conditions by genomewide association study. PLoS Genet. 13:e1006889. doi: 10.1371/journal.pgen. 1006889 
Li, Y., Zeng, X.-F., Zhao, Y.-C., Li, J.-R., and Zhao, D.-G. (2017). Identification of a new rice low-tiller mutant and association analyses based on the slaf-seq method. Plant Mol. Biol. Rep. 35, 72-82. doi: 10.1007/s11105-016-1002-2

Liang, H., Yu, Y., Yang, H., Xu, L., Dong, W., Du, H., et al. (2014). Inheritance and QTL mapping of related root traits in soybean at the seedling stage. Theor. Appl. Genet. 127, 2127-2137. doi: 10.1007/s00122-014-2366-Z

Liang, Q., Cheng, X., Mei, M., Yan, X., and Liao, H. (2010). QTL analysis of root traits as related to phosphorus efficiency in soybean. Ann. Bot. 106, 223-234. doi: $10.1093 / \mathrm{aob} / \mathrm{mcq} 097$

Libault, M., Farmer, A., Brechenmacher, L., Drnevich, J., Langley, R. J., Bilgin, D. D., et al. (2010). Complete transcriptome of the soybean root hair cell, a single-cell model, and its alteration in response to Bradyrhizobium japonicum infection. Plant Physiol. 152, 541-552. doi: 10.1104/pp.109.148379

Lippert, C., Listgarten, J., Liu, Y., Kadie, C. M., Davidson, R. I., and Heckerman, D. (2011). FaST linear mixed models for genome-wide association studies. Nat. Methods 8:833. doi: 10.1038/nmeth.1681

Liu, X., Huang, M., Fan, B., Buckler, E. S., and Zhang, Z. (2016). Iterative usage of fixed and random effect models for powerful and efficient genomewide association studies. PLoS Genet. 12:e1005767. doi: 10.1371/journal.pgen. 1005767

Liu, X., Qin, D., Piersanti, A., Zhang, Q., Miceli, C., and Wang, P. (2020). Genomewide association study identifies candidate genes related to oleic acid content in soybean seeds. BMC plant Biol. 20:399. doi: 10.1186/s12870-020-02607-w

Liu, Y., Gai, J., and Lu, H. (2005a). Identification of rhizosphere abiotic stress tolerance and related root traits in soybean [Glycine max (L.) Merr.]. Zuo Wu Xиe Bao 31, 1132-1137.

Liu, Y., Gai, J. Y., Lu, H. N., Wang, Y. J., and Chen, S. Y. (2005b). Identification of drought tolerant germplasm and inheritance and QTL mapping of related root traits in soybean (Glycine max (L.) Merr.). Acta Genet. Sin. 32, 855-863.

Livak, K. J., and Schmittgen, T. D. (2001). Analysis of relative gene expression data using real-time quantitative PCR and the $2-{ }^{\triangle} \triangle C T$ method. Methods 25, 402-408. doi: 10.1006/meth.2001.1262

Lü, C., Guo, J., Wang, Y., Leng, J., Yang, G., Hou, W., et al. (2010). Identification, inheritance analysis and QTL mapping of root and shoot traits in soybean variety PI471938 with tolerance to wilting. Acta Agron. Sin. 36, 1476-1483.

Lü, H., Yang, Y., Li, H., Liu, Q., Zhang, J., Yin, J., et al. (2018). Genome-wide association studies of photosynthetic traits related to phosphorus efficiency in soybean. Front. Plant Sci. 9:1226. doi: 10.3389/fpls.2018.01226

Manavalan, L. P., Prince, S. J., Musket, T. A., Chaky, J., Deshmukh, R., Vuong, T. D., et al. (2015). Identification of novel QTL governing root architectural traits in an interspecific soybean population. PLoS One 10:e120490. doi: 10. 1371/journal.pone. 0120490

McKenna, A., Hanna, M., Banks, E., Sivachenko, A., Cibulskis, K., Kernytsky, A., et al. (2010). The genome analysis toolkit: a MapReduce framework for analyzing next-generation DNA sequencing data. Genome Res. 20, 1297-1303.

Murray, M., and Thompson, W. (1980). Protocol of DNA isolation. Nucleic Acids Res. 8, 4321-4325. doi: 10.1093/nar/8.19.4321

Nye, P. H., and Tinker, P. B. (1977). Solute Movement in the Soil-Root System. Berkeley, CA: Univ of California Press.

Pace, J., Gardner, C., Romay, C., Ganapathysubramanian, B., and Lübberstedt, T. (2015). Genome-wide association analysis of seedling root development in maize (Zea mays L.). BMC Genomics 16:47. doi: 10.1186/s12864-015$1226-9$

Pandey, M. K., Agarwal, G., Kale, S. M., Clevenger, J., Nayak, S. N., Sriswathi, M., et al. (2017). Development and evaluation of a high density genotyping 'Axiom_Arachis' array with $58 \mathrm{~K}$ SNPs for accelerating genetics and breeding in groundnut. Sci. Rep. 7:40577. doi: 10.1038/srep 40577

Pantalone, V. R., Burton, J. W., and Carter, T. E. (1996). Soybean fibrous root heritability and genotypic correlations with agronomic and seed quality traits. Crop Sci. 36, 1120-1125.

Price, A. L., Patterson, N. J., Plenge, R. M., Weinblatt, M. E., Shadick, N. A., and Reich, D. (2006). Principal components analysis corrects for stratification in genome-wide association studies. Nat. Genet. 38:904. doi: 10.1038/ng1847

Prince, S. J., Valliyodan, B., Ye, H., Yang, M., Tai, S., Hu, W., et al. (2019). Understanding genetic control of root system architecture in soybean: Insights into the genetic basis of lateral root number. Plant Cell Environ. 42, 212-229. doi: $10.1111 /$ pce. 13333
Prince, S. J., Vuong, T. D., Wu, X., Bai, Y., Lu, F., Kumpatla, S. P., et al. (2020). Mapping quantitative trait loci for soybean seedling shoot and root architecture traits in an inter-specific genetic population. Front. Plant Sci. 11:1284. doi: $10.3389 /$ fpls.2020.01284

Purcell, S., Neale, B., Todd-Brown, K., Thomas, L., Ferreira, M. A., Bender, D., et al. (2007). PLINK: a tool set for whole-genome association and population-based linkage analyses. Am. J. Hum. Genet. 81, 559-575. doi: 10.1086/519795

Qiu, L.-J., Chen, P.-Y., Liu, Z.-X., Li, Y.-H., Guan, R.-X., Wang, L.-H., et al. (2011). The worldwide utilization of the Chinese soybean germplasm collection. Plant Genet. Resour. 9, 109-122. doi: 10.1017/S1479262110000493

Riedelsheimer, C., Lisec, J., Czedik-Eysenberg, A., Sulpice, R., Flis, A., Grieder, C., et al. (2012). Genome-wide association mapping of leaf metabolic profiles for dissecting complex traits in maize. Proc. Natl. Acad. Sci. U.S.A. 109, 8872-8877. doi: 10.1073/pnas.1120813109

Rong, Z., Hai-Feng, C., Xian-Zhi, W., Bao-Duo, W., Shui-Lian, C., Zhang, X.-J., et al. (2011). Analysis of QTLs for root traits at seedling stage in soybean. Acta Agron. Sin. 37, 1151-1158.

Rosa, A., Diaz, D., Hansel, F., Sebastian, J., and Adee, E. (2019). Genotypic variation on root growth and nutrient uptake in corn and soybean. Agrosyst. Geosci. Environ. 2, 1-12.

Sattelmacher, B., Klotz, F., and Marschner, H. (1990). Influence of the nitrogen level on root growth and morphology of two potato varieties differing in nitrogen acquisition. Plant Soil 123, 131-137. doi: 10.1007/BF00011258

Seck, W., Torkamaneh, D., and Belzile, F. (2020). Comprehensive genome-wide association analysis reveals the genetic basis of root system architecture in soybean. Front. Plant Sci. 11:590740. doi: 10.3389/fpls.2020.590740

Severin, A. J., Woody, J. L., Bolon, Y.-T., Joseph, B., Diers, B. W., Farmer, A. D., et al. (2010). RNA-Seq Atlas of Glycine max: a guide to the soybean transcriptome. BMC Plant Biol. 10:160. doi: 10.1186/1471-2229-10-160

Sinclair, T. R. (2011). Challenges in breeding for yield increase for drought. Trends Plant Sci. 16, 289-293. doi: 10.1016/j.tplants.2011.02.008

Smith, S., and De Smet, I. (2012). Root system architecture: insights from Arabidopsis and cereal crops. Philos. Trans. R. Soc. Lond. B Biol. Sci. 367, 1441-1452. doi: 10.1098/rstb.2011.0234

Suji, K., Prince, K. S. J., Mankhar, P. S., Kanagaraj, P., Poornima, R., Amutha, $\mathrm{K}$., et al. (2012). Evaluation of rice (Oryza sativa L.) near iso-genic lines with root QTLs for plant production and root traits in rainfed target populations of environment. Field Crops Res. 137, 89-96. doi: 10.1016/j.fcr.2012.08.006

Sun, X., Liu, D., Zhang, X., Li, W., Liu, H., Hong, W., et al. (2013). SLAF-seq: an efficient method of large-scale de novo SNP discovery and genotyping using high-throughput sequencing. PLoS One 8:e58700. doi: 10.1371/journal.pone. 0058700

Tamura, K., Peterson, D., Peterson, N., Stecher, G., Nei, M., and Kumar, S. (2011). MEGA5: molecular evolutionary genetics analysis using maximum likelihood, evolutionary distance, and maximum parsimony methods. Mol. Biol. Evol. 28, 2731-2739. doi: 10.1093/molbev/msr121

Thomson, M. J. (2014). High-throughput SNP genotyping to accelerate crop improvement. Plant Breed. Biotechnol. 2, 195-212. doi: 10.9787/PBB.2014. 2.3.195

Tian, P. (1984). Ecotypes of root system in soybean cultivars. Acta Agron. Sin. 10, 173-178.

Tran, L.-S. P., and Mochida, K. (2010). Functional genomics of soybean for improvement of productivity in adverse conditions. Funct. Integr. Genomics 10, 447-462.

Tuberosa, R., and Salvi, S. (2007). "From QTLs to genes controlling root traits in maize," in Scale and Complexity in Plant Systems Research: Gene-Plant-Crop Relations, eds J. H. J. Spiertz, P. C. Struik, and H. H. van Laar (Wageningen: Springer), 13-22. Frontis.

Van Nguyen, L., Takahashi, R., Githiri, S. M., Rodriguez, T. O., Tsutsumi, N., Kajihara, S., et al. (2017). Mapping quantitative trait loci for root development under hypoxia conditions in soybean (Glycine max L. Merr.). Theor. Appl. Genet. 130, 743-755. doi: 10.1007/s00122-016-2847-3

Wang, H.-L., Yu, D.-Y., Wang, Y.-J., Chen, S.-Y., and Gai, J.-Y. (2004). Mapping QTLs of soybean root weight with RIL population NJRIKY. Yi Chuan 26, 333-336.

Xu, X., Xu, R., Zhu, B., Yu, T., Qu, W., Lu, L., et al. (2015). A high-density genetic map of cucumber derived from Specific Length Amplified Fragment sequencing (SLAF-seq). Front. Plant Sci. 5:768. doi: 10.3389/fpls.2014.00768 
Yang, N., Lu, Y., Yang, X., Huang, J., Zhou, Y., Ali, F., et al. (2014). Genome wide association studies using a new nonparametric model reveal the genetic architecture of 17 agronomic traits in an enlarged maize association panel. PLoS Genet. 10:e1004573. doi: 10.1371/journal.pgen.10 04573

Yang, S., Chen, J., He, X., Yu, D., and Gai, J. (2005). Inheritance of drought tolerance and root traits of seedling in soybeans. Soybean Sci. 24, 275-280.

Yang, X., Yan, J., Shah, T., Warburton, M. L., Li, Q., Li, L., et al. (2010). Genetic analysis and characterization of a new maize association mapping panel for quantitative trait loci dissection. Theor. Appl. Genet. 121, 417-431. doi: 10.1007/ s00122-010-1320-y

Ying, L., Jun-yi, G., and Hui-neng, L. (2007). Genetic variation of root traits at seedling stage and their relationship with stress tolerance in soybean. Soybean Sci. 26, 127-133.

Zeng, A., Chen, P., Korth, K., Hancock, F., Pereira, A., Brye, K., et al. (2017). Genome-wide association study (GWAS) of salt tolerance in worldwide soybean germplasm lines. Mol. Breed. 37:30. doi: 10.1007/s11032-017-0634-8

Zhang, H., Fan, X., Zhang, Y., Jiang, J., and Liu, C. (2017). Identification of favorable SNP alleles and candidate genes for seedlessness in Vitis vinifera L. using genome-wide association mapping. Euphytica 213:136. doi: 10.1007/ s10681-017-1919-Z

Zhang, W.-K., Wang, Y.-J., Luo, G.-Z., Zhang, J.-S., He, C.-Y., Wu, X.-L., et al. (2004). QTL mapping of ten agronomic traits on the soybean (Glycine max L. Merr.) genetic map and their association with EST markers. Theor. Appl. Genet. 108, 1131-1139. doi: 10.1007/s00122-003-1527-2

Zhang, X., Zhang, J., He, X., Wang, Y., Ma, X., and Yin, D. (2017). Genome-wide association study of major agronomic traits related to domestication in peanut. Front. Plant Sci. 8:1611. doi: 10.3389/fpls.2017. 01611

Zhang, Y., Wang, L., Xin, H., Li, D., Ma, C., Ding, X., et al. (2013). Construction of a high-density genetic map for sesame based on large scale marker development by specific length amplified fragment (SLAF) sequencing. BMC Plant Biol. 13:141. doi: 10.1186/1471-2229-13-141

Zhang, Z., Ersoz, E., Lai, C.-Q., Todhunter, R. J., Tiwari, H. K., Gore, M. A., et al. (2010). Mixed linear model approach adapted for genome-wide association studies. Nat. Genet. 42:355. doi: 10.1038/ng.546
Zhao, K., Tung, C.-W., Eizenga, G. C., Wright, M. H., Ali, M. L., Price, A. H., et al. (2011). Genome-wide association mapping reveals a rich genetic architecture of complex traits in Oryza sativa. Nat. Commun. 2:467. doi: 10.1038/ncomms 1467

Zheng, Z., Sun, Z., Fang, Y., Qi, F., Liu, H., Miao, L., et al. (2018). Genetic diversity, population structure, and botanical variety of 320 global peanut accessions revealed through tunable genotyping-by-sequencing. Sci. Rep. 8:14500. doi: 10.1038/s41598-018-32800-9

Zhou, Q., Zhou, C., Zheng, W., Mason, A. S., Fan, S., Wu, C., et al. (2017) Genome-Wide SNP markers based on SLAF-Seq uncover breeding traces in rapeseed (Brassica napus L.). Front. Plant Sci. 8:648. doi: 10.3389/fpls.2017. 00648

Zhou, R., Wang, X., Chen, H., Zhang, X., Shan, Z., Wu, X., et al. (2009). QTL analysis of lodging and related traits in soybean. Acta Agron. Sin. 35, 57-65. doi: 10.3724/SP.J.1006.2009.00057

Zhou, Z., Jiang, Y., Wang, Z., Gou, Z., Lyu, J., Li, W., et al. (2015). Resequencing 302 wild and cultivated accessions identifies genes related to domestication and improvement in soybean. Nat. Biotechnol. 33:408. doi: 10.1038/nbt.3096

Conflict of Interest: The authors declare that the research was conducted in the absence of any commercial or financial relationships that could be construed as a potential conflict of interest.

Publisher's Note: All claims expressed in this article are solely those of the authors and do not necessarily represent those of their affiliated organizations, or those of the publisher, the editors and the reviewers. Any product that may be evaluated in this article, or claim that may be made by its manufacturer, is not guaranteed or endorsed by the publisher.

Copyright (C) 2021 Mandozai, Moussa, Zhang, Qu, Du, Anwari, Al Amin and Wang. This is an open-access article distributed under the terms of the Creative Commons Attribution License (CC BY). The use, distribution or reproduction in other forums is permitted, provided the original author(s) and the copyright owner(s) are credited and that the original publication in this journal is cited, in accordance with accepted academic practice. No use, distribution or reproduction is permitted which does not comply with these terms. 